\title{
Pseudokinases: update on their functions and evaluation as new drug targets
}

The pseudokinase complement of the human kinase superfamily consists of approximately 60 signaling proteins, which lacks one or more of the amino acids typically required to correctly align ATP and metal ions, and phosphorylate protein substrates. Recent studies in the pseudokinase field have begun to expose the biological relevance of pseudokinases, which are now thought to perform a diverse range of physiological roles and are connected to a multitude of human diseases, including cancer. In this review, we discuss how and why members of the 'pseudokinome' represent important new targets for drug discovery, and describe how knowledge of protein structure and function provides informative clues to help guide the rational chemical design or repurposing of inhibitors to target pseudokinases.

First draft submitted: 3 November 2016; Accepted for publication: 7 December 2016; Published online: 18 January 2017

Keywords: cancer $\bullet$ cysteine $\bullet$ cysteinome $\bullet$ disease $\bullet$ enzyme $\bullet$ inhibitor $\bullet$ kinase $\bullet$ kinome - pseudoenzyme $\bullet$ pseudokinase $\bullet$ signaling

\section{Background}

Protein pseudokinases are ubiquitous among the kingdoms of life, accounting for approximately $10 \%$ of a typical vertebrate kinome $[1-$ 4], increasing to $>50 \%$ of the kinome in the unicellular protist Giardia [5]. Their catalytically active protein kinase counterparts are well-known regulators of many eukaryotic cellular processes, which tightly control intracellular signaling network by facilitating the catalytic transfer of phosphate from ATP to substrate proteins. With approximately $50 \%$ of all proteins thought to be phosphorylated in cells [6], it is not surprising that dysregulation of protein kinases is frequently a driving factor (or consequence) in a variety of human medical disorders including cancers [7,8], Type 2 diabetes [9], cardiovascular [10], neurodegenerative [11] and developmental diseases [12]. This has led to a considerable effort to develop small-molecule therapeutic agents to control aberrant protein phosphorylation, with notable early successes including Ima- tinib to inhibit BCR-ABL1 chronic myelogenous leukemia [13], vemurafanib to target V600E $B R A F$-mutant melanoma [14] and Lapatinib to treat ERBB2/HER2-amplified tumors [15]. Chemical structures of some of the kinase inhibitors discussed in this review are depicted in Figure 1.

In stark contrast, pseudokinases are much less-well characterized. They are traditionally defined as pseudoenzymes [16] lacking one or more of the canonical amino acids or motifs that are typically required to efficiently coordinate ATP and transfer phosphate in catalytically active kinase counterparts $[3,17,18]$. Although a loss of catalytic activity appears to be a feature of many pseudokinases, the ability to bind to and/or hydrolyze ATP (sometimes very weakly) can be detected in a number of cases [19], including CASK [20,21], TRIB2 [22], JAK2 [23], HER3 [24,25], withno-lysine kinase (WNK) [26], STRAD $\alpha$ [27], MLKL [28] and kinase suppressor of ras 1 and $2(\mathrm{KSR} 1 / 2)$ [29]. A retained affinity for ATP
Dominic P Byrne1, Daniel M Foulkes ${ }^{1} \&$ Patrick A Eyers $*, 1$ 'Department of Biochemistry, Institute of Integrative Biology, University of Liverpool, Liverpool, UK *Author for correspondence: patrick.eyers@liverpool.ac.uk 
indicates that nucleotide-dependent switching mechanisms may have been preserved in the fold in order to regulate a proportion of pseudokinase-dependent signaling, either driven through catalytic activity or by ATP-driven conformational transitions. Together, these requirements for signaling highlight pseudokinases as an important emerging class of drug targets [30]. Indeed, ATP binding is now understood to be essential for the biological activities of noncatalytic pseudokinases such as STRAD $\alpha$ [27] and HER3 [31]. In contrast, several pseudokinases that possess a highly degraded ATP-binding site (such as vaccinia-related kinase 3 [VRK3]) appear to have lost the ability to bind ATP completely [4,19]. It is therefore now becoming clear that the rate-limiting cellular outputs of protein kinases, and in particular pseudokinases, are not restricted to intrinsic catalytic turnover [32]. This suggests that the development of ATP competitive, covalent or allosteric small molecules to modulate phosphorylation-independent cellular signaling by kinases and pseudokinases represents a new pharmacological challenge.

\section{Disease-associated pseudokinases}

Approximately two-thirds of pseudokinases have been implicated in a very diverse range of human diseases [30]. In the following section, we briefly discuss important disease-associated pseudokinases, whose biological activities might be susceptible to therapeutic intervention with small-molecule ligands.

\section{Janus kinase-, hybrid kinase- and pseudokinase-signaling polypeptides}

The JAK family of nonreceptor tyrosine kinases consist of JAK1, JAK2, JAK3 and TYK2 [33], which share seven regions of sequence termed Janus homology (JH) domains. JH1 consists of a conventional tyrosine kinase domain that becomes activated upon stimulation of type I/II cytokine receptors [33], and is involved in a variety of biological processes including hematopoiesis and the regulation of the immune system [34]. The JH1 domain is usually negatively regulated by $\mathrm{JH} 2$, a pseudokinase-containing domain that lacks the catalytic Asp residue in the HRD motif, which is typically required for catalytic transfer of the $\gamma$-phosphoryl group to the alcoholic substrate, but nonetheless still appears to regulate JAK2 signaling through ATP binding and/or weak catalytic activity [35]. In fact, JAK2 amino acid residues Ser523 and Tyr570 are potential substrate sites for the low activity $\mathrm{JH} 2$ pseudokinase domain, although targeted inactivation of the $\mathrm{JH} 1$ domain completely abolishes Tyr570 phosphorylation in vitro, suggesting a rate-limiting requirement for $\mathrm{JH} 1$ in this process [35-37]. Despite binding to ATP, the JH2 pseudokinase domains of JAK1 and TYK2 appear to be catalytically inactive, and neither carry conserved JH1 Ser523 and Tyr570 residues, potentially suggestive of a lack of phosphorylation-driven autoregulation [38,39]. However, ATP binding (which stabilizes the JH2 domain of TYK2 without significant structural reorganization) is still thought to serve a critical allosteric function in maintaining the tyrosine kinase domain in an auto-inhibited state [38]. The critical importance of $\mathrm{JH} 2$-dependent modulation of JAK kinase function is further emphasized by the marked enhancement in kinase activity upon deletion of the pseudokinase domains of JAK2 and $3[36,40]$.

To date, JAKs have perhaps been the most intensively studied of the pseudokinases and this can be directly attributed to the occurrence of multiple disease-driving somatic mutations in JH2 domains [34]. Of particular note is a prominent gain of function oncogenic variant of JAK2 (V617F) [41-44] that has been strongly implicated in multiple myeloproliferative neoplasms [39] and is used diagnostically to guide therapeutic intervention strategies owing to its occurrence in approximately $95 \%$ of polycythemia vera and approximately $60 \%$ of essential thrombocythemia and primary myelofibrosis cases $[43,45]$. The consequence of this amino acid substitution is hyperactivation of JAK2 and constitutive downstream signaling due to a presumed destabilization of the auto-inhibitory JH1-JH2 interaction $[46,47]$. Interestingly, ATP binding to the pseudokinase domain of JAK2 was recently shown to be essential for the hyperactivation phenotype of pathogenic JH2 mutants such as V617F [48]. This clear association with human malignancies, combined with the documented ATP-mediated regulation of the pseudokinase domain, suggests that $\mathrm{JH} 2$ domains might be suitable small-molecule drug targets for the treatment of cancer and auto-immune diseases. In this regard, ruxolitinib, an orally available tyrosine kinase inhibitor that targets the JAK1 and JAK2 JH1 canonical kinase domains, was the first US FDA approved inhibitor for the treatment of myelofibrosis [49], and has also been approved for patients with polycythemia vera to control thrombotic events that fail to respond to hydroxurea [50]. Suppression of JAK signaling could also be achieved indirectly with small molecules that promote $\mathrm{JH} 2$ auto-inhibition of JH1 kinase output, which has already been suggested mechanistically for TYK2.

\section{HER3/ErbB3 pseudokinases}

The clinically significant pseudokinase HER 3 belongs to the epidermal EGFR family of receptor tyrosine kinases. HER3 is a pseudokinase due to a lack of the canonical catalytic Asp residue [17]. In spite of this, HER3 is able to bind tightly to ATP (Kd 1 $\mu \mathrm{M})$, 
and although it also exhibits very low tyrosine kinase activity [24], it appears unlikely that this vestigial phosphotransferase capacity is required for HER3 function in cells where this has been tested [25,51]. Instead, ligand-induced heterodimerization of HER3 with EGFR (HER1) or HER2 stimulates allosteric transactivation of these kinases and modulates a myriad of cellular responses, including downstream activation of the PI3K/AKT signaling pathway [52,53]. HER3 is also required for malignant progression in HER2amplified breast cancers [54], which originally led to the idea that it might be targeted by conventional kinase inhibitors. Indeed, HER3 overexpression and mutation is associated with a multitude of human cancers [55,56] and it also represents a potential diagnostic marker in breast cancer, where it is amplified in around $60 \%$ of cases [57]. One of the major consequences of aberrant HER3 activation is the hyperactivation of PI3K/AKT signaling, which classically drives prosurvival pathways, and has been linked to the development of multidrug resistance in some cancers [58]. This is supported by the finding that antibody-mediated dual targeting of HER3 and EGFR might be a useful method to overcome acquired resistance elicited towards EGFR-targeted therapeutics [59]. Interestingly, several cancer-associated somatic mutations that confer a gain-of-function phenotype for HER3 have also been reported $[55,60]$ and structure-based studies indicate that enhancement of HER3 allosteric activation function may underscore the molecular mechanisms of these 'activating' mutations [61]. In this regard, inhibitor compounds that specifically target the active signaling state of HER 2 in the HER2-HER3 oncogenic complex [51] might have therapeutic potential.

\section{STRAD $\alpha$}

The catalytically inactive pseudokinase STRAD $\alpha$ (and the closely-related, but enigmatic, pseudokinase STRAD $\beta$ ) contains a severely degraded kinase domain, which is devoid of canonical $\beta 3$ Lys, DFG and HRD motif residues. STRAD $\alpha$ functions as an allosteric regulator of the tumor-suppressor kinase LKB1, and its biological function is dependent on the adoption of a closed 'active' conformation, which is generated upon cooperative binding to ATP and the auxillary scaffolding protein MO25 $\alpha$ [27,62]. Formation of the LKB1-STRAD $\alpha-\mathrm{MO} 25 \alpha$ heterotrimeric complex results in LKB1-dependent phosphorylation of AMPK and the modulation of many cellular proliferation signaling pathways [27,63]. Several human cancers, and the rare inherited disease Peurtz-Jeghers syndrome, are the consequence of loss of function mutations in LKB1 that perturb binding and activation by STRAD $\alpha$ and MO25 $\alpha[62,64]$. Furthermore, the effectiveness of the broad antitumor drug metformin appears to be contingent on an ability to stimulate LKB1-dependent activation of AMPK [65], which positions STRAD $\alpha$, as an important potential therapeutic target for the manipulation of downstream effectors of LKB1.

\section{Kinase suppressor of Ras 1 \& 2}

KSR1 and 2 are important scaffolding pseudokinase proteins that coordinate the formation of the (oncogenic) Raf-MEK-ERK signaling complex [66,67] and are also important regulators of immune function and metabolism [68]. KSR 1 and 2 lack the canonical $\beta 3$ Lys but are proposed to have dual scaffolding and catalytic roles, with established phosphorylated protein substrates including MEK1 [29,69] and C-RAF-1 [70,71] within this complex. However, the weak kinase activity of mammalian KSRs is likely dispensable for MEK phosphorylation and MAPK signaling in kinase-impaired mutants [72], which challenges the physiological importance of KSR catalytic activity. KSR1 is a prospective therapeutic target of Ras-driven tumors due to its oncogenic potential in human cells $[67,73,74]$. Disease associated mutations in KSR2 that disrupt KSR2 signaling through the Raf-MEK-ERK pathway have also been linked to obesity, insulin resistance and impaired cellular fuel oxidation [75].

\section{MLKL}

Despite lacking a Glycine-rich loop, and possessing noncanonical HGK and GFE residues in place of the typical DFG and HRD motifs, mixed lineage kinase domain like (MLKL) binds robustly to ATP in an obligate cation-independent manner. However, it appears unable to catalyze ATP hydrolysis [28,76], instead driving necroptosis (a form of regulated cell death) in a catalytically independent manner. MLKL function is indispensable for TNF- $\alpha$-induced necroptosis, which proceeds via the obligate phosphorylation of two activation loop residues (Thr357 and Ser358) in MLKL by the canonical upstream kinase RIP3 [76,77]. Phosphorylation at these residues stabilizes the active conformation of MLKL, which facilitates release of the $\mathrm{N}$-terminal domain four-helix bundle that drives MLKL oligomerization, membrane translocation and subsequent activation of necroptosis [78]. Under normal physiological conditions, necroptosis is triggered in response to physiological or pathophysiological stimuli as part of an innate defense against pathogens that suppress apoptosis [79]. However, deregulation of necroptosis is a risk factor in a multitude of autoimmune and inflammatory diseases [80], highlighting MLKL as a potential novel drug target. So far, only the biological 
functions of MLKL in relation to necroptosis have been dissected, but a mutation $(\mathrm{E} 351 \mathrm{~K})$ in the nonconventional GFE motif (which has evolved in place of the DFG motif) has also been described in human cancers, and could possibly be linked to an altered affinity or usage for nucleotides [28,81].

\section{Tribbles pseudokinases}

Three human Tribbles (TRIB) homologs (TRIB1, TRIB2 and TRIB3) are characterized by an N-terminal PEST region, a pseudokinase domain containing an atypical DFG metal-binding motif (E[S/N]LED) and a C-terminal COP1-binding region [22,82]. TRIB proteins are important regulators of many divergent cellular processes including lipoprotein metabolism, immune function and cellular differentiation and proliferation [83]. This is achieved via two major modes of action; the first involving the E3-ligase-dependent ubiquitination of their protein substrates, and the second by modulation of MAPK and AKT signaling modules [82,84-87]. The recently solved crystal structure of TRIB1 reveals how the SLE motif adopts a unique 'inactive'-like conformation that obstructs nucleotide binding in the TRIB1 ATP-binding site and potentially also represents a physical barrier to ATP-competitive inhibitors [88]. This adaption presumably helps TRIB1 to function as an efficient regulator of ubiquitin-mediated substrate degradation and limits or rules out ATP binding and hydrolysis. In contrast, TRIB2 and 3 demonstrate very weak ATP affinity and ATP hydrolysis in vitro [22]. Dysregulation of TRIB proteins has been implicated in a variety of human cancers, including acute myeloid leukemia (AML) [89,90], lung [91], hepatic cancers [92] and melanoma [93]. In the case of TRIB2, these malignancies are primarily thought to proceed via TRIB-mediated degradation of the C/EBP $\alpha$ transcription factor. Interestingly, TRIB2 requires an intact nucleotide-binding site in order to drive AML in mice [89], which presents a potential opportunity for pharmacological intervention with compounds that target the TRIB2 ATPbinding site. In this regard, it is noteworthy that our lab has recently identified small-molecule ligands from a library of kinase inhibitors that interact with TRIB2, although the precise binding modes have yet to be characterized [82].

As well as being linked to AML, TRIB1 has been identified in Down's syndrome-related human acute megakaryocytic leukemia [94]. In this case, the cancerassociated TRIB1 mutant, R107L, enhances the degradation of $\mathrm{C} / \mathrm{EBP} \alpha$ through an unknown mechanism. Although TRIB3 does not modulate the stability of $\mathrm{C} / \mathrm{EBP} \alpha$ it has been implicated in the degradation of acetyl-CoA carboxylase [95]. In addition TRIB3 has a role in glucose-induced insulin resistance in diabetic rats [96].

\section{WNK}

The WNK family of Ser/Thr pseudokinases (comprising WNK1-4) [97] are defined because they lack the conserved $\beta 3$ lysine, which was long assumed to be indispensable for nucleotide binding and stabilization of the active kinase conformation [26]. Despite this apparent handicap, WNK family kinases readily demonstrate phosphorylation-dependent regulation of a host of intracellular substrates. This innovation is made possible by the evolution a novel mechanism of catalysis; the terminal residue in the glycine-rich loop (often a Gly in kinases) is conserved as a Lys residue in WNKs and this residue provides the compensatory charge to support the binding of ATP [26]. It has been proposed that the site normally occupied by the $\beta 3$ lysine functions as a chloride sensor by binding to halide ions and inhibiting WNK activation by autophosphorylation, thus facilitating a feedback mechanism for the regulation of $\mathrm{Cl}^{-}$levels [98]. Consistently, in its active state, WNKs phosphorylate and activate SPAK and the oxidative stress response kinase (OSR1), which in turn modulate the downstream activities of $\mathrm{Na}+$-driven, $\mathrm{Cl}^{-}$-importing NKCCs $\left(\mathrm{Na}+/ \mathrm{K}+/ \mathrm{Cl}^{-}\right.$ cotransporters) in order to modulate blood pressure and ion homeostasis [97].

WNK family members are differentially expressed in tissues and the deregulated function of neuronal WNK isoforms has been implicated in various diseases, including hereditary neuropathy and glioma [97]. In addition, mutations in WNK1 and WNK4 have been linked to hereditary hypertension [99]. WNK1 and WNK3 also stimulate proliferative and invasive activity in glioma cells [100-102]. Conversely, WNK2 predominantly plays a role as a tumor suppressor and downregulates cellular proliferation by increasing and decreasing activity of Rac1 and RhoA respectively [103].

\section{Protein kinase \& pseudokinase small- molecule inhibitors}

The majority of protein kinase inhibitors are reversible ATP-competitive molecules that can broadly be classified into two major groups: type 1 inhibitors, which bind to the ATP-binding sites of targets assuming a closed 'active' conformation (where the $\alpha \mathrm{C}$ helix and DFG motif are in an inward orientation) and type II inhibitors that bind to- and lock kinases in a more open 'inactive' state by occupying a hydrophobic groove adjacent to the ATP-binding site that only becomes accessible in the 'DFG-out' conformation [104]. Achieving selectivity among the large kinase superfamily, which all share a high degree of 
sequence conservation in the active site, has proven extremely challenging, and is only truly possible for ATP-competitive inhibitors through the exploitation of unique features found in regions surrounding the ATP-binding site. For example, the pyridinyl-imdazole SB 203580 was an early example of a partially selective type I inhibitor that targets p38 MAPK $\alpha$ and $\beta$ isoforms, but not the very closely related $\gamma$ or $\delta$ isoforms, and this specificity among MAP kinase isoforms was deduced to be absolutely dependent on the presence of a bulky 'gatekeeper' Met in the latter, which imparts steric hindrance to inhibitor binding [105,106]. In actual fact, the use of promiscuous inhibitors that simultaneously target multiple dysregulated signaling pathways might actually be advantageous from an oncological perspective [107], but clearly potentially increases the risk of adverse side-effects. Such compounds are also much less practical for the treatment of (noncancer) diseases, where logical mechanistic approaches have historically been preferable, reflecting the likelihood for long-term therapeutic maintenance and a high therapeutic index. In this regard, specific targeting of pseudokinases may indeed be useful, in large part because these proteins as a class characteristically possess unusual amino acid compositions and structural architecture in the ATP-binding site, be it functional or not $[18,30]$. Exploiting these evolutionary differences might facilitate the generation of highly specific ligands that are directed against pseudokinases, especially those whose regulatory functions are linked to adaptions around the nucleotide-binding site. Alternatively, chemical genetic 'bump-and-hole' approaches, such as those pioneered by Shokat and colleagues [108] might be useful approaches for swiftly evaluating pseudokinase signaling with modified kinase inhibitors after mutagenesis of the pseudokinase gatekeeper [22,109].

\section{Type I \& II ATP-competitive inhibitors: insights from kinase structures}

Currently, it is estimated that approximately $40 \%$ of the pseudokinome retains the ability to interact with adenine-based nucleotides, and most, if not all, of these proteins might theoretically be modulated by ATP-competitive inhibitors [19,110]. To test this assertion, two highly generic kinase inhibitors, DAP and/or VI16832, were shown to bind to nearly a third of a sample set of human pseudokinases [19], building upon the very limited small-molecule interactions described for pseudokinases, which with the exception of ErbB3/HER3 have often been omitted from biochemical or cellular screening approaches. Indeed, the pan-tyrosine kinase inhibitor Bosutinib (SKI-606) was one of the first examples of a clinically approved multikinase ATP-mimetic compound capable of mod- ulating the function of the HER3, which it binds to with subnanomolar affinity [111,112]. A low nanomolar HER3 affinity for other kinase inhibitors, including familiar clinical compounds such as Dasatinib, and the covalent compound Neratinib, have also been described in the literature [112]. In addition, HER3 kinase activity has been successfully targeted with compounds such as Compound 2 (Figure 1) [51], or shown to bind to ATP-competitive compounds such as KIN001-051 [25]. Paradoxically, binding of bosutinib to the ATP-binding pocket of HER3 actually increases the capacity of the pseudokinase to operate as an allosteric activator of the 'receiver' kinase EGFR in a heterodimer, most likely by stabilizing the 'active' HER3 conformation [111]. Although in this specific example bosutinib fails to elicit a phenotypic 'inhibitory response', the data clearly support the hypothesis that pseudokinases can be targeted (and perhaps regulated) by ATP-competitive ligands. This finding also highlights the potential risk (or advantage, depending upon one's point of view) of small molecules behaving as allosteric activators depending on the binding mode and induced effects to the kinase fold [113]. This phenomenon has previously been observed for catalytically active kinases such as B-RAF, for which binding of certain inhibitors stabilizes its active state and promotes homo- and hetero-dimerization (with C-RAF), stimulating MAPK kinase signaling in the process [114,115]. In contrast, inhibitors such as Vemurafenib that stabilize an inactive conformation do not promote B-RAF/CRAF heterodimerization [114]. As discussed previously, the adoption of a ligand-induced 'active' conformation in STRAD $\alpha$ is a critical step in the allosteric activation of LKB1 tumor suppressor function [62]. Development of compounds that lock STRAD $\alpha$ in an appropriate conformation might therefore be an effective therapeutic strategy to inhibit downstream proliferative pathways in specific tumors.

The TYK2 pseudokinase domain is also likely to be amenable to similar targeting by ATP-competitive therapeutics, as the TYK2 JH2 domain has recently been cocrystalized in complex with small-molecule inhibitors that stabilize the intradomain auto-inhibitory interaction with the tyrosine kinase domain, and block downstream signaling cascades [38,116]. Although currently scarce, pseudokinases whose biological functions are linked to ATP hydrolysis and direct substrate phosphorylation, such as WNK, are also obvious targets for traditional ATP-competitive inhibitors. As discussed previously, KSR2 exhibits dual scaffold and catalytic activity, and this latter functionality can be modulated by pan kinase inhibitors such as ASC24, which greatly reduces KSR2-dependent phosphorylation of MEK1 at non-BRAF phosphorylated Ser resi- 


\section{Bosutinib (HER3)}

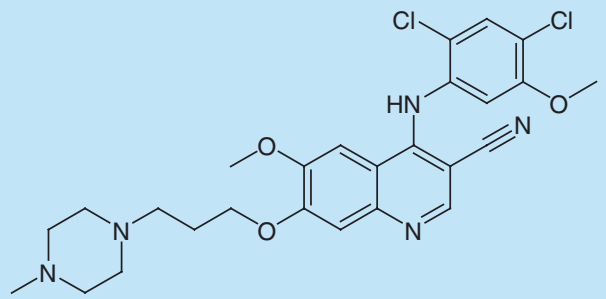

Lapatinib (HER2)<smiles>CS(=O)(=O)CCNCc1ccc(-c2ccc3ncnc(Nc4ccc(OCc5cccc(F)c5)c(Cl)c4)c3c2)o1</smiles>

Neratinib (HER2 covalent)<smiles>C#CC(=O)Nc1ccc2c(c1)/C(=C/c1[nH]c(CC)nc1C)C(=O)N2</smiles>

JH295 (NEK2 covalent)<smiles>C#CC(=O)Nc1ccc2c(c1)/C(=C/c1[nH]c(CC)nc1C)C(=O)N2</smiles>

Dasatinib (HER3)

HO<smiles>CCN1CCN(c2cc(Nc3ncc(C(=O)Nc4c(C)cccc4Cl)s3)nc(C)n2)CC1</smiles>

Ibrutinib (BTK covalent)

Afatinib (HER2 covalent)

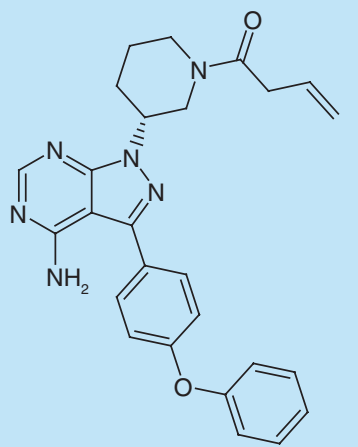

FIIN-1 (FGF1-3 covalent)<smiles>C=CC(=O)Nc1cccc(CN2C(=O)N(c3c(Cl)c(OC)cc(OC)c3Cl)Cc3cnc(CCCCCN(CC)CC)nc32)c1</smiles><smiles>C=CC(=O)Nc1cccc(C)c1Nc1ncc2cc(-c3c(Cl)c(OC)cc(OC)c3Cl)ccc2n1</smiles>

\section{BLU9331 (FGFR4 covalent)}

VI6832 (broad pseudokinase)

THZ531 (CDK12/13 covalent)<smiles>NCCOc1ccc(Nc2ncc3ccc(=O)n(C4CC5CCC4C5)c3n2)cc1</smiles> 


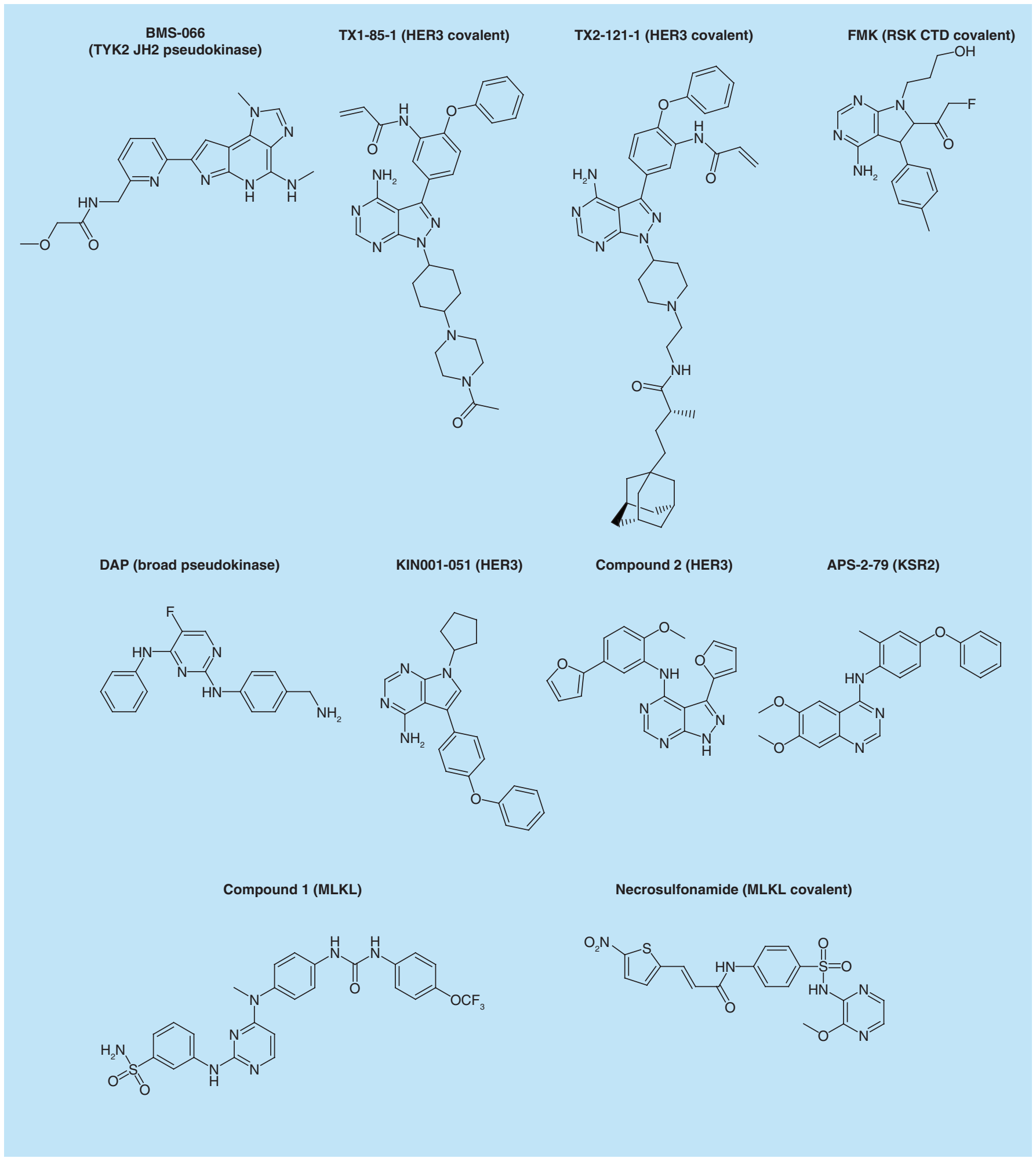

Figure 1. Chemical structure of selected ATP-competitive and covalent kinase and pseudokinase small-molecule inhibitors (cont. from facing page). Major kinase and pseudokinase targets are shown, where these have been reported in the literature. Several covalent compounds that target Cys residues in kinases or pseudokinase are noted. 
dues [29]. However, considering the scant evidence for physiological catalytic outputs from pseudokinases such as KSR and JAK JH2 domains, the effectiveness of this approach may be limited.

Although mitigation of aberrant pseudokinase signaling through type- 1 inhibition is a potentially attractive prospect, realizing target selectivity will nearly always be a barrier for effective cellular analysis using chemical biology approaches [109], and certainly more so than for many clinical evaluations, where efficacy and safety can often trump the need for detailed mechanistic understanding. The pursuit of type II kinase inhibitors might therefore be an alternative approach to achieve greater target specificity, especially considering the reduced amino acid conservation in the areas immediately surrounding the ATP pocket (which only becomes exposed in the DFG-out state) and also the greater conformational variability that is observed between kinases in their inactive states [117]. The development of compounds that trap or block these different induced conformations is a strategy that was exploited to a great effect in the development of imatinib, which stabilizes ABL in the DFG-out conformation $[118,119]$ and is approved for the treatment of patients with BCR-ABL positive chronic myeloid leukemia $[120,121]$. Importantly, the extent to which pesudokinase activity is regulated by dynamic conformational transitions is not yet fully resolved. This notwithstanding, many of the hydrophobic amino acid residues that control the conformations of canonical kinases are also conserved in numerous pseudokinases, which predicts that an ability to adopt some form of regulatory active and/or inactive state upon nucleotide or ligand binding has at least been partially retained [122]. For example, crystal structures of RNase L, which contains a catalytically inactive pseudokinase domain [123-125], reveal a rigid closed structure that nonetheless bears similarity to the canonical kinase 'DFG-in' fold. Of potential regulatory relevance, this architecture is stabilized when bound to the nonhydrolyzable ATP analog AMP-PNP, but becomes severely disrupted in the absence of nucleotides, reverting to an inactive conformation. Back-to-back homodimerization and consequently activation of the RNase domain was shown to be entirely dependent on nucleotide binding and the adoption of the closed conformation. It is also predicted that non-enzymatic activity of MLKL is dependent on a conformational change to an active form brought about by RIPK3-mediated phosphorylation of the activation loop [76]. Catalytic-independent STRAD $\alpha$ signaling also requires a conformational transition to an active 'closed' state that is induced by ATP binding and through complex formation with the MO25 scaffold protein [27]. Based on these and other examples, it is apparent that the noncatalytic activities of many pseudokinases may be closely linked to an active 'DFG-in' conformation, and inhibitors that select for or stabilize inactive conformations could potentiate phosphorylation independent signaling networks. As discussed previously, Bosutinib behaves as an allosteric activator of HER3, which would seem to indicate that HER3 signaling is at least partially dependent on conformational transition states [111]. It seems reasonable to hypothesize that compounds that sequester HER3 as an inactive monomer could begin to display the qualities of bona fide inhibitors. In support of this, antibodies that trap HER3 in an inactive conformation or directly inhibit its association with active kinase family members neutralize several facets of HER3 dependent signaling [126,127]. Recently, an ATP-competitive inhibitor (APS-2-79) of KSR2 was shown to stabilize the pseudokinase in an inactive state and modulate KSR2-dependent MAPK signaling by antagonizing RAF heterodimerization in a manner independent of KSR2 catalytic activity [128]. Interestingly, MEK phosphorylation by KSR2 is also stimulated by an RAF-mediated allosteric transition of KSR2 suggesting that both the catalytic and scaffold output of the protein could be pharmacologically targeted [29]. Molecular switching of MLKL to a pseudoactive state is a determining factor for its oligomerization and thus membrane translocation, which drives necroptosis. Necroptosis was inhibited by a small ATPcompetitive molecule, termed compound 1, although it is not clear if this occurs via the stabilization of an inactive or active state [129], and the target specificity and mechanism of action have recently been re-evaluated [130]. To our knowledge, the only example of a molecule driving type II inhibition with a pseudokinase is that described for KSR2. However, this important study serves to expose the potential vulnerability of the pseudokinome in general to small-molecule intervention. It is also worth considering that stabilization of a 'native' DFG-out state is not necessarily the only route via which inhibition can be achieved, as exemplified by various classes of ATP-competitive inhibitors that induce an unusual inactive conformation in Aurora A that is not associated with 'normal' catalytic function $[131,132]$.

\section{Type III 'allosteric' inhibitors}

Two additional modes of kinase inhibition have also been described; type III inhibitors that bind to sites outside of the ATP-binding pocket to drive conformational changes, and type IV (covalent) cysteine-driven interactions. These compounds can offer a distinct efficacious and selective advantage over noncovalent type I and II inhibitors, which target highly conserved regions of the 
ATP-binding site, and under physiological conditions have limited potency in the presence of high intracellular concentrations of ATP [133]. In contrast, type III kinase inhibitors exert their (allosteric) effects either by indirectly targeting catalytic activity or by modulating noncatalytic function, and generally achieve excellent target precision through binding to less conserved, non-ATP-binding sites [134]. For example, GNF-5 binds to the myristate pocket of $\mathrm{ABL}$ and inhibits the kinase by allosterically inducing a restructuring of the ATPbinding site [135]. In contrast, AKT can be locked in an inactive conformation by compounds which bind at the interface between the kinase domain and the pleckstrin homology domain [136,137], and PDK1 can be targeted by allosteric ligands binding in the PIF-binding pocket in the kinase $\mathrm{N}$-lobe that communicates with the ATP-binding site when occupied [138]. Unfortunately, most allosteric inhibitors appear to have been discovered serendipitously, and the rational design of new target-specific compounds requires a detailed structural and biomolecular understanding of the protein and its regulatory mechanisms. Indeed, only approximately $25 \%$ of mammalian pseudokinase domains have been structurally characterized (Table 1). Although no proof of concept for type III inhibition currently exists in the public domain for pseudokinases, the growing number of pseudokinases observed to undergo 'kinase-like' conformational changes in response to appropriate cellular cues is a promising indication of the potential of this area for biomedical research. Indeed, the interesting effects of ATP-competitive allosteric regulators of KSR2 on distinct inhibitors of MEK signaling in RAS-mutant cancer cells lends further credence to this theory [128].

\section{Covalent kinase inhibitors \& the human protein kinome cysteinome}

The majority of covalent 'type IV' kinase inhibitors are versions of established kinase inhibitors that have undergone structure-guided modification, specifically with the addition of an electrophilic 'warhead' to target the nucleophilic thiol groups of cysteine residues found in their targets [150]. Originally thought to lack clinical utility, in part due to issues with promiscuity, compounds containing warheads that target Cys residues have seen a resurgence [151,152], driven by the approval of the nonspecific Bruton's tyrosine kinase inhibitor Ibrutinib [153] and the discovery of more potent and selective, compounds such as acalabrutinib (ACP-196) [154]. Although Cys residues encoded in the human kinome have not been reported to serve a direct catalytic role, in marked contrast to protein phosphatases, they are still found in and around the ATP pocket in relatively high abundance, providing opportunities for redox regulation of the catalytic output or protein interactomes of a large proportion of protein kinases $[155,156]$. Indeed, based on structuraland sequence-based analysis, it has previously been estimated that as many as $39 \%$ of protein kinases have accessible noncatalytic cysteines in their active sites $[150,157]$ and there has been considerable effort dedicated to developing inhibitors that block access to the ATP pocket by covalently modifying such residues. Covalent inhibitors have several desirable characteristics compared with reversible inhibitors, especially in instances where target specificity can be increased, or perhaps even guaranteed, based on the distribution of Cys residues in the kinase domain. The reactions are energetically favorable, requiring lower inhibitor concentrations to achieve high efficacy, and once bound the covalent molecule provides terminal inhibition, meaning new protein synthesis must occur to restore kinase function. Importantly, because targeted Cys residues are not uniformly conserved among kinases and pseudokinases, they act as specificity filters to limit inhibitor promiscuity. Moreover, with deeper analysis of kinase conformational changes, thiol groups that are differentially exposed in active and inactive kinase states will likely produce a diverse and versatile reservoir of rather specific drug targets [150]. From a clinical perspective, one potential downside of using irreversible inhibitors is unanticipated toxicity from concentration dependent off-target adduct formation with hyper-reactive cysteines of unrelated proteins, an occurrence that will have to be evaluated on a case-by-case basis $[133,158,159]$. The following section describes how our understanding of kinase structures has guided the design of irreversible covalent inhibitors with useful target specificity, and how this might be applied as a framework for the design of similar compounds directed against pseudokinases.

The term 'cysteinome' describes a convenient methodology for the categorization of targetable cysteine loci in and around the ATP pocket (Figure 2) [157]. To date, most attention has been directed to the development of compounds that target Cys on the hinge region amino acids that connect the $\mathrm{N}$ - and C-terminal kinase lobes. For example, the noncatalytic hinge-region cysteine (Cys481) of Bruton's tyrosine kinase is the target of Ibrutinib [160], an irreversible inhibitor which is used for the treatment of B-cell malignancies chronic lymphocytic leukemia [161] and mantle cell lymphoma [162]. Sequence alignment reveals that all four other TEC family kinases (BMX, ITK, TEC and TXK), in addition to BLK, JAK3, MKK7 and ErbB family members (EGFR, HER2 and HER4), possess an equivalent cysteine at this position, and display cross-reactive liabilities toward certain compounds [157,159]. As discussed earlier, the JAKs are highly attractive therapeutic targets for the treatment of numerous human diseases, but development of compounds with strong selectivity within this family has been impeded by the extremely high sequence 


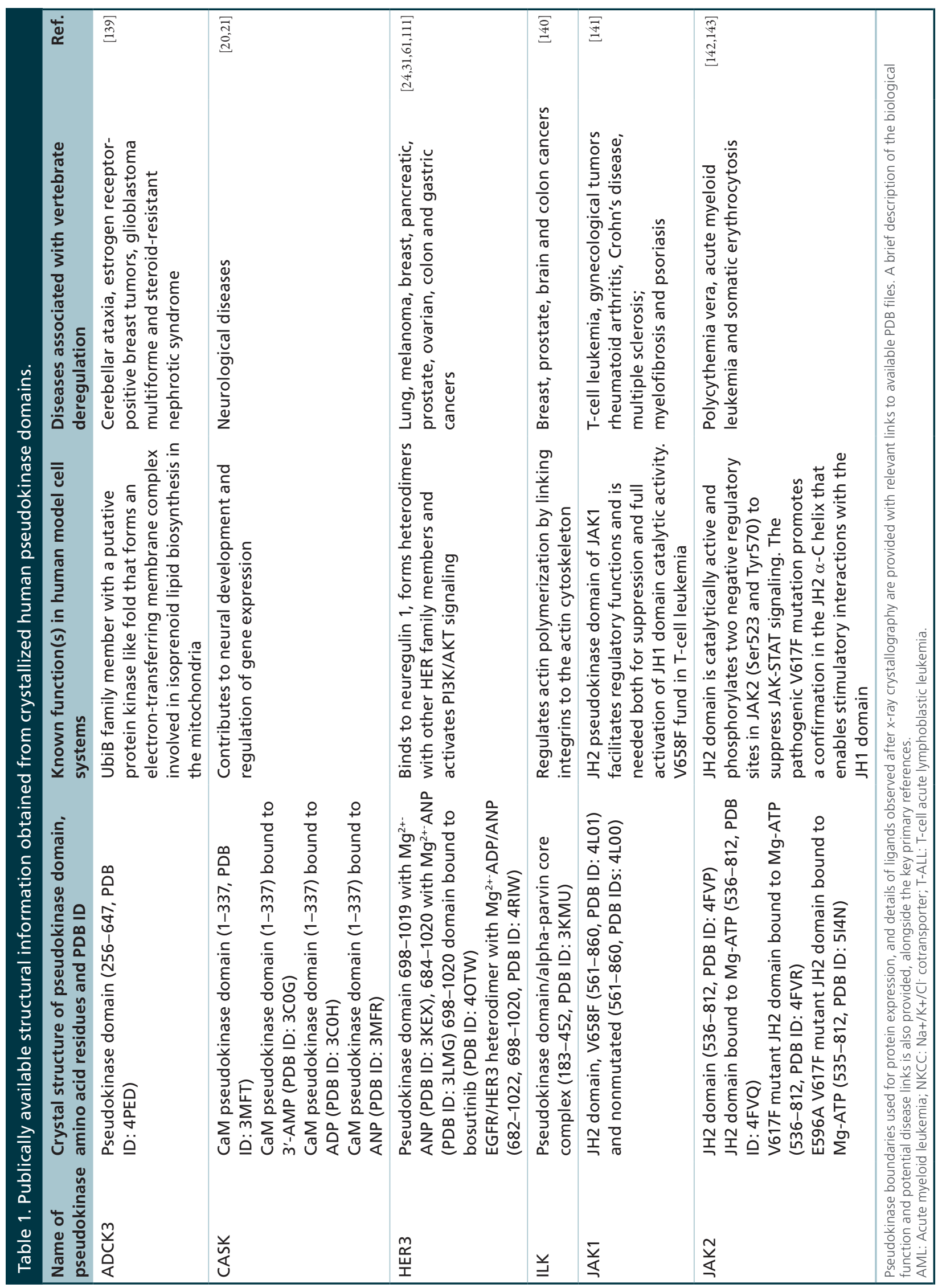




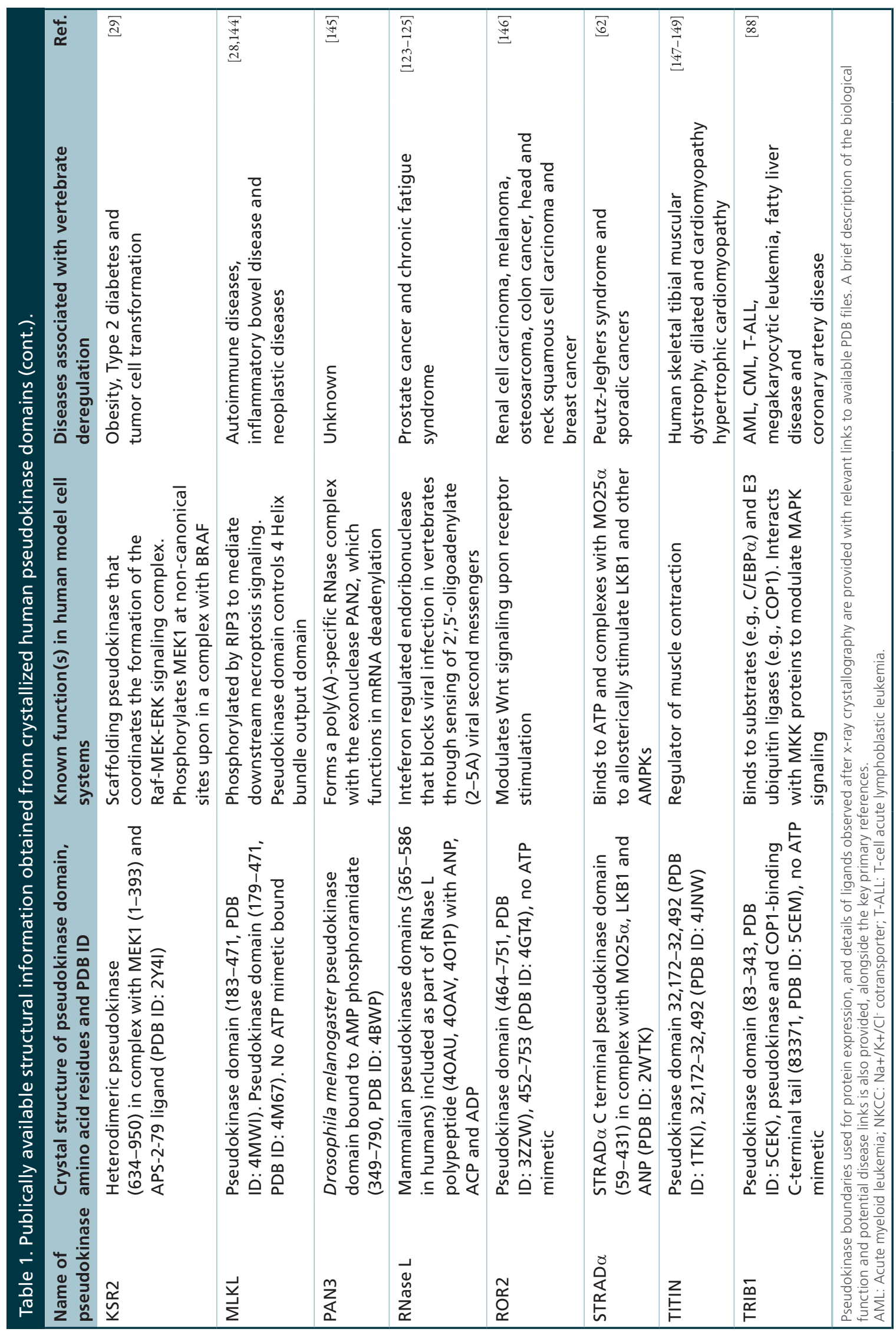




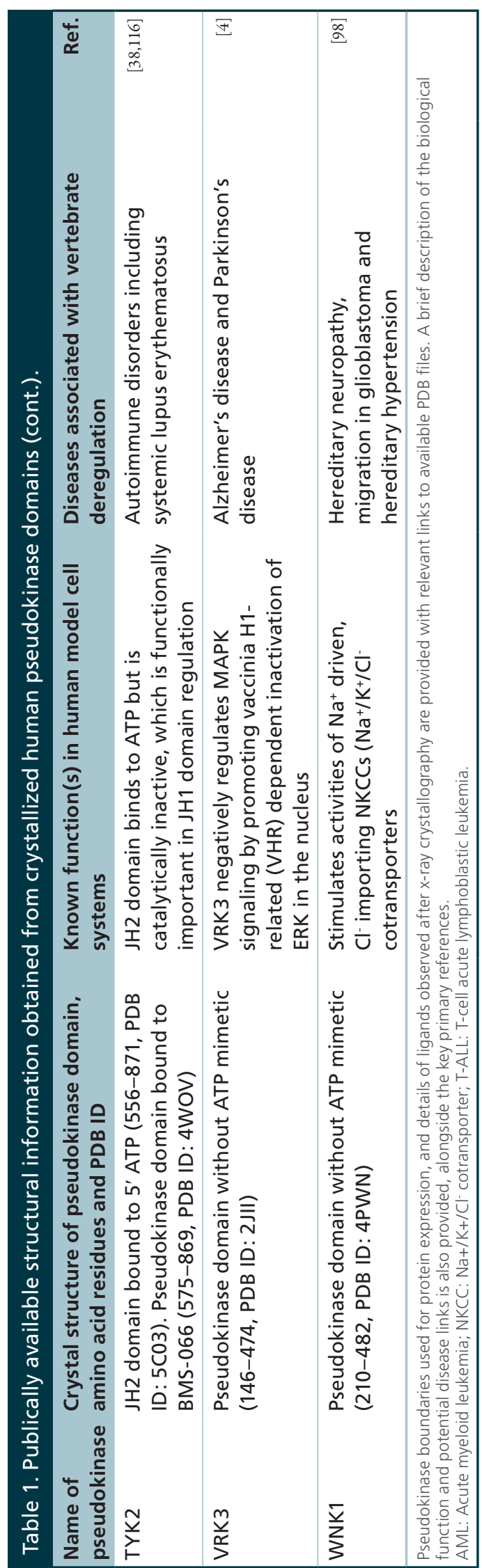

identity shared across their conserved tyrosine kinase active sites. The hinge region around Cys909 of the JAK3 JH1 (kinase) domain is unique among JAKs, and provided a potential selectivity handle to develop irreversible inhibitor compounds which specifically target JAK3 with little cross-reactivity with other JAK kinases or kinases carrying analogous Cys residues [163]. The equivalent cysteine (Cys797) of EGFR is also of clinical significance, and is covalently targeted by clinically approved covalent inhibitors such as Afatanib for the treatment of non-small-cell lung cancer [164]. Interestingly, a nonconserved and extremely rare hinge-region cysteine was exploited to design highly specific inhibitors against EphB3. Only two other human kinases, LKB1 and PINK1, share this residue and in these cases the Cys appears less accessible compared with that of EphB3, which likely greatly improves the specificity of the compound [165]. A highly potent covalent inhibitor of JNK family kinases also targets a conserved cysteine on the hinge-loop region (JNK 1/2 Cys 116, JNK 3 Cys 154) [166]. FGFR4 contains another unique hinge region cysteine (Cys552) that is not conserved among the three other human FGFR paralogs and is rare $(>1 \%)$ among human kinases in general. Hagel et al., developed BLU9931, a covalent compound with $\geq 50$-fold greater inhibition of FGFR 4 as compared with FGFR1-3, and which exhibited significant antitumor activity in hepatocellular carcinoma xenograft models [167]. Curiously, all FGFRs carry a Cys on the glycine-rich loop that is the target of the potent irreversible pan-FGFR inhibitor, FIIN-1, which was developed from the noncovalent inhibitor PD173074 [168]. To our knowledge FIIN-1 is the only current example of a covalent inhibitor that targets a cysteine at this specific position, although many kinases (and pseudokinases) including PLKs, RSKs and ZAK also have cysteines on their glycine-rich loops [157].

Cysteine amino acids situated on the 'roof region' of the ATP-binding pocket, immediately following the glycine-rich loop, can also be accessible for covalent modification. Cohen et al., exploited such a Cys to target p90 ribosomal protein S6 kinase (RSK) family members with the compound FMK [169]. In the design of FMK, Cohen et al., took advantage of a small gatekeeper Thr (residue 463) found in RSK to ensure selectivity over kinases with analogous Cys residues, but that contain bulky gatekeeper residues that are prohibitive to compound binding. Cys22 of human centrosomal kinase, NEK2, is located at an identical position to that of the RSK family of kinases and is targeted by oxindole propynamide 16 (JH295), which irreversibly inhibits NEK2 cellular activity [170]. Deregulation of the pseudokinase HER3 is a feature of several cancers [171] and the protein was initially thought to be 'undruggable' as it lacked detectable kinase activity despite binding ATP [24]. How- 
ever, TX2-121-1-adamantane conjugates form a covalent bond with Cys 721 on the roof of the HER3 ATPbinding pocket, and partially blocks HER3-dependent signaling by targeting the protein for degradation in some HER3-dependent cell lines [25].

A specific Cys residue adjacent to the DFG motif is another possible target for covalent modification, and one whose solvent accessibility might be regulated through conformational fluctuations in pseudokinase structures. However, this Cys residue is present in about $10 \%$ of all human kinases, which decreases the likelihood of obtaining acceptable levels of selectivity [133]. In agreement, the natural product cis-enone covalent inhibitor hypothemycin was shown to bind to $>90 \%$ of tested kinases carrying this conserved Cys residue, including ERK1, MEK1/2, PDGFRs, FLT3 and VEGFRs [172]. Even in this extreme case, careful structure-guided design facilitated the generation of a compound which could discriminately target VEGFR-2 over proteins bearing analogous Cys residues [173]. This serves to demonstrate the influence that alternate chemical scaffolds can have on an inhibitors ability to achieve adequate selectivity, even when targeting highly conserved binding sites. Sequence analysis of the pseudokinase NOK reveals that it also contains a Cys at this position [18]. Interestingly, there are only two canonical human protein kinases containing a Cys residue at the gatekeeper position ( $\mathrm{SgK} 494$ and MOK), neither of which have been targeted covalently, and based on our analysis no human pseudokinases.

Cys residues located outside the ATP-binding pocket on the activation loop are also potential targets for smallmolecule intervention. However, the challenge of structure-guided design of such compounds is exacerbated by the inherent flexibility of this region. Interestingly, Cys covalent modification of the activation segment by biologically active factors that induce allosteric inhibition have already been described for several canonical kinases $[155,156,174,175]$ which suggest that covalent targeting of the activation loop may be a viable prospect. Unfortunately, much like allosteric inhibitors, most nonATP-binding site compounds have been discovered by chance, which reduces the ability to identify suitable modifiable-thiol groups. Recently however, the rationale design of an irreversible inhibitor of CDK12 and 13 that targets a Cys remote from the kinase domain has been reported [53]. THZ531 achieves CDK12 and CDK13 inhibition by covalently binding to a $\mathrm{C}$-terminal cysteine residue and making additional contacts with the ATP pocket via its flexible linker region. Even more surprising was the discovery that MLKL activity could also be regulated by a covalent compound that exerted its function by binding to a region distal to the active site. Cys86 on the N-terminal coiled coil domain of MLKL was cova- lently modified by necrosulfonamide, an interaction that blocked necroptosis in human cells [77]. This presents the exciting opportunity to scrutinize non-ATP located Cys residues (including outside the pseudokinase domain) for covalent modification and may greatly expand the number of potential drug targets.

\section{The human pseudokinase cysteinome}

The examples discussed in this review serve to demonstrate the surprising levels of selectivity that can be achieved by drugs that target nonconserved thiol groups, many of which are also found among the human pseudokinases. To date, most studies discussing the protein kinase 'cysteinome' have precluded atypical kinases and pseudokinases from evaluation, and only Cys 721 in the pseudokinase domain of HER3 (Figure 2) and a nonpseudokinase domain cysteine of MLKL have (knowingly) been targeted by a covalent compound up to now [25,77]. For this reason, we have analyzed the frequency and distribution of Cys residues among the complete set of kinase and pseudokinase domains in the human kinome, using new comparative Kinview software [176] and PKA nomenclature to standardize amino acid positions (Figure 3). Cys residues were identified at over 100 distinct pseudokinase domain loci (some 273 Cys residues in total). Interestingly, some Cys positions were observed at high frequency in both pseudo and canonical kinases (e.g., Cys at equivalent position 273), whereas other Cys residues were less evenly distributed between the two groups. For example, Cys at position 200 (within the canonical activation segment) occurs in approximately $20 \%$ of protein kinases, but a Cys was identified in just two pseudokinases (PSKH2 and VACAMKL/CAMKV). Cys-200 has known regulatory functions among canonical kinases including PKA, partly due to its close proximity to activating sites of auto-phosphorylation [175]. We therefore predict that Cys underrepresentation at this position in pseudokinases is a likely consequence of their loss of regulatable catalytic function. In marked contrast, Cys at PKAequivalent positions 169, 228 and 268 are found at near tenfold higher frequency in pseudokinases than among conventional kinases (Figure 3, asterisks). Of particular interest is a Cys amino acid at position 169, which is found in $14 \%$ of pseudokinases, but less than $2 \%$ of canonical kinases and lies within the extended HRD motif, which is classically involved in phosphate transfer in active kinases. This residue might therefore be a useful target for pseudokinase covalent modification. Of note, a hinge region Cys residue that has previously been targeted by a covalent inhibitor of FGFR4 is also present in the pseudokinase Sgk495 (Cys 123, PKA numbering, corresponding to Sgk495 Cys 143). SgK495 is a highly degraded 'orphan' pseudokinase most closely related to 


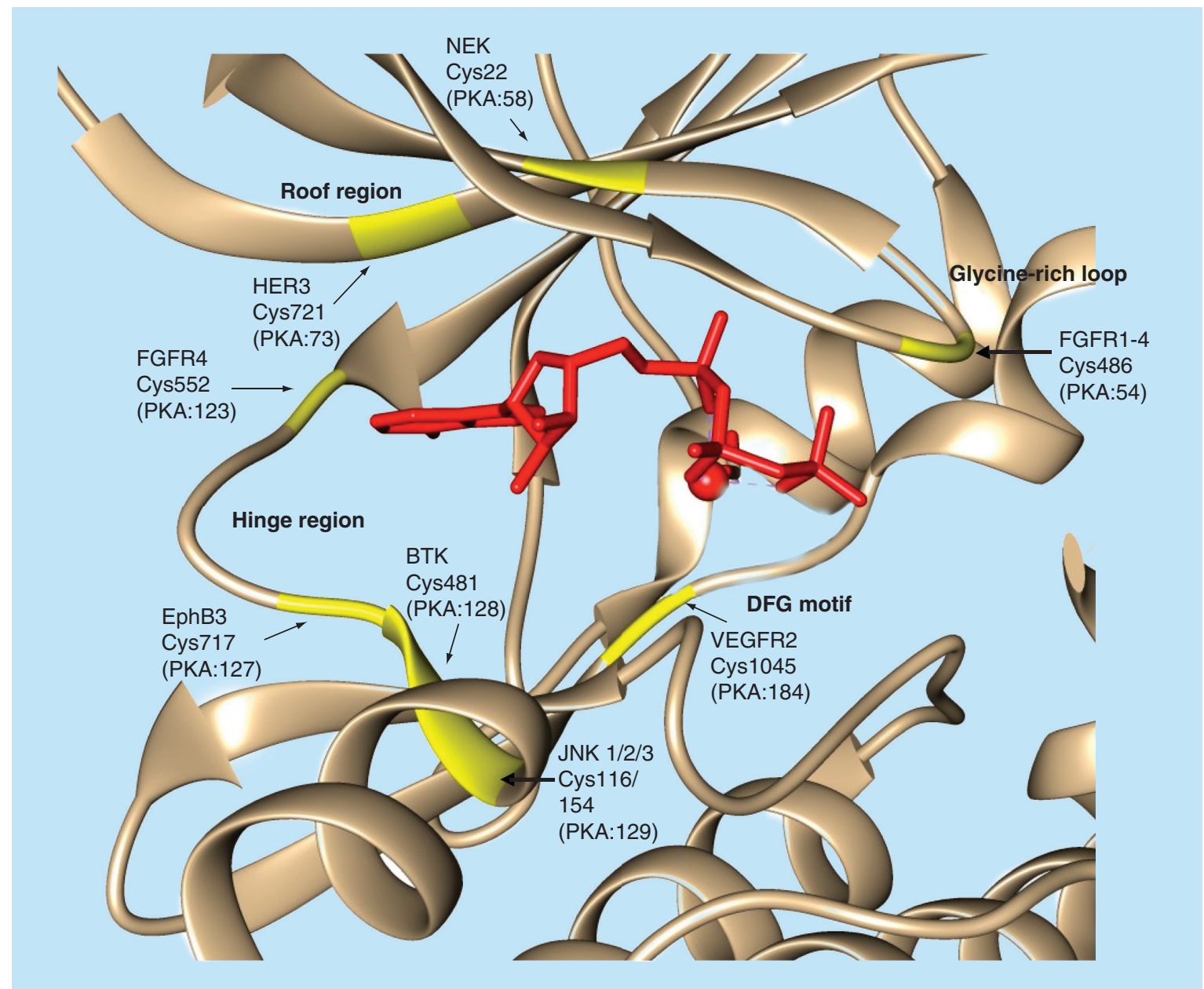

Figure 2. Schematic representation of positional distribution at key positions among the targeted protein human kinome 'cysteinome'. Yellow residues indicate positions of Cys residues that have been targeted for covalent modification in protein kinases and pseudokinases, and modelled using the HER3 tyrosine kinase structure (PDB ID: 3LMG). The actual site of covalent attachment, and the PKA equivalent residue, is also shown. AMP-PNP ligand is shown in red.

the Tribbles subfamily of pseudokinases [22]. Although our simple analysis does not take in to account the thiol side-chain solvent accessibility of Cys residues, it clearly demonstrates that these residues are present, potentially biologically relevant, and potentially targetable by appropriate chemical reagents in human pseudokinases. They could therefore be evaluated much more closely as new molecular targets for small molecules related to classical kinase inhibitors. One obvious avenue for drug screening, perhaps based on simple biophysical measurements or cell-based coupling, might include focused libraries comprised known inhibitors, including those with designed covalent bond forming capabilities (Figure 1).

\section{Future perspective}

Pseudokinases evolved unique signaling mechanisms to regulate a broad range of cellular processes, many of which go awry in disease. It might therefore make sense to develop novel medicinal chemistry approaches to treat pathophysiological conditions by interfering with pseudokinase-mediated signaling. The past decade has seen a huge expansion in our understanding of various aspects of pseudokinase structure and function, which has presented new opportunities to interrogate their function with small-molecule inhibitors. Although this review has primarily focused on inhibitors that directly (or indirectly) interfere with potential pseudokinase transitional states, there is tremendous scope for inhibitors with alternative modes of action, such as those that might sterically interfere with pseudokinase-driven protein-protein interactions. In this regard, stapled peptides might be considered to disrupt pseudokinase interactions, as illustrated by the development of stapled peptides that impair AKAP-mediated localization of the PKA complex in an isoform-selective manner $[177,178]$. Indeed, although pseudokinases frequently possess zero or vestigial catalytic activity, they can still form functional complexes with active kinases, often facilitating a 
crucial layer of allosteric regulation. By interfering with this process, pseudokinase function might indirectly be modulated with compounds that preferentially target the induced active-state of binding partners, as exemplified by inhibitors directed against the HER2-HER3 oncogenic pairing [51,61]. A final, potentially significant, challenge if pseudokinase therapeutics are to succeed is the likely development of drug resistance, a scourge associated with the structurally dynamic kinase domain fold. Whether the innate dynamic conformational plas-

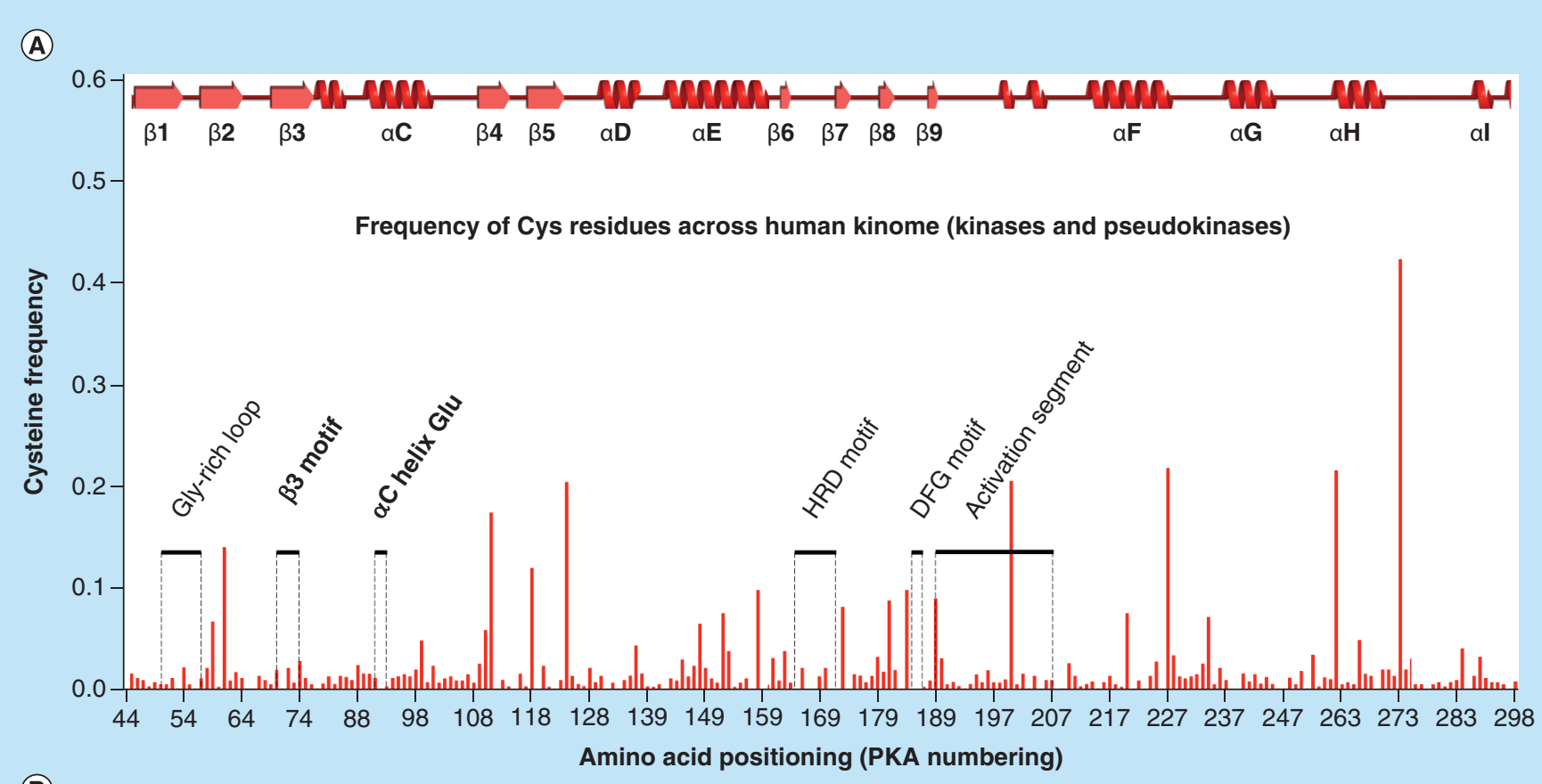

(B)

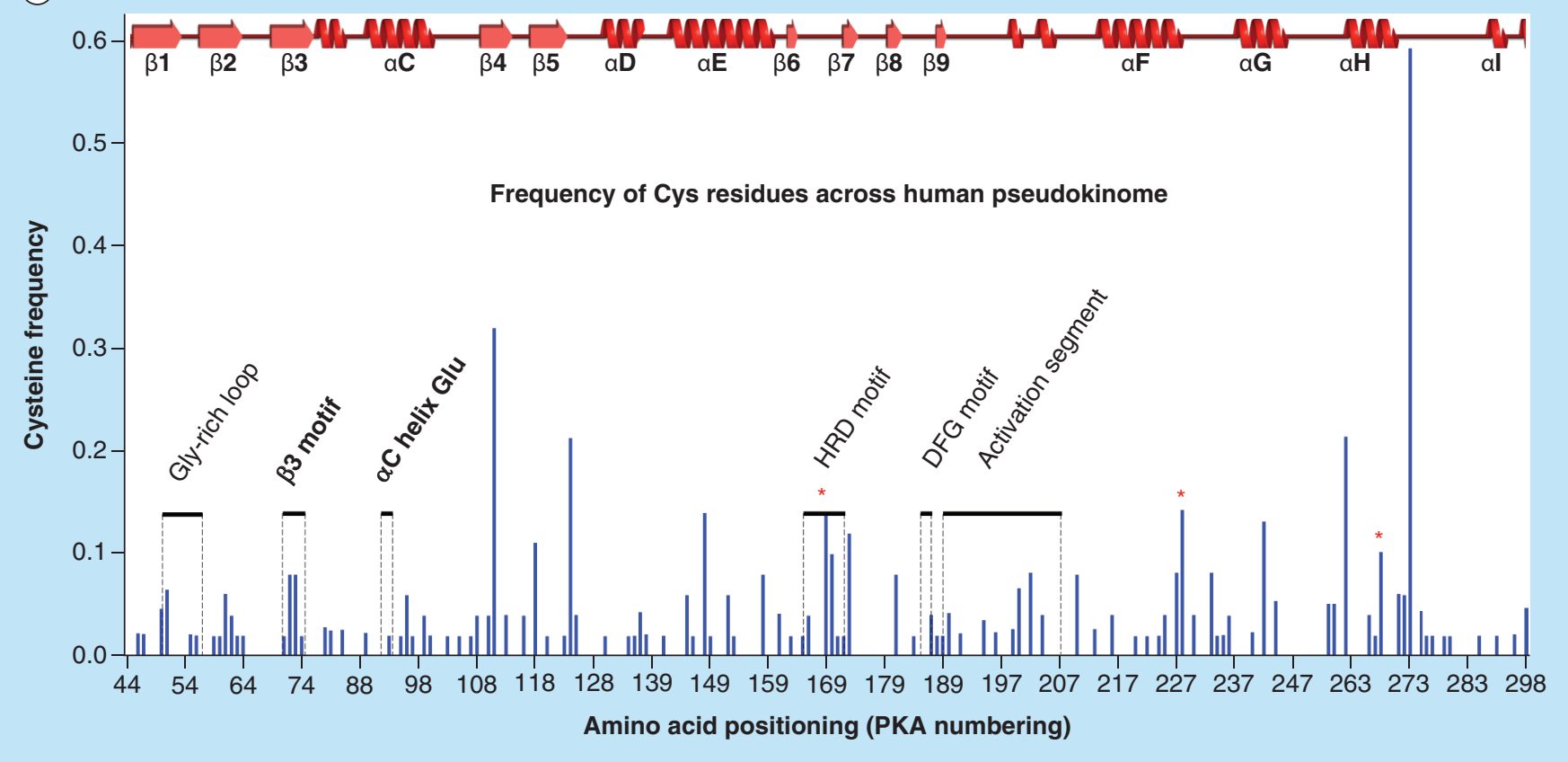

Figure 3. Frequency and distribution of cysteine amino acids found within the human kinome. The protein kinase ontology (ProKinO) browser was used to perform an integrative analysis across all human kinase and pseudokinase sequences (the kinome) in order to reveal Cys-residue frequencies and their linear positions relative to the PKA kinase domain. (A) Frequency and distribution of cysteine residues in all human kinases. (B) Frequency and distribution of cysteine residues in the human pseudokinases. The relative position of secondary structure elements for the canonical PKA kinase domain is shown in both panels, with canonical catalytic and regulatory motifs highlighted. Asterisks distinguish positions where a particularly high frequency of cysteine residues are present in pseudokinases, which if chemically labile, represent potential targets for chemical covalent modification. 
ticity of pseudokinases, which has only recently been appreciated, underpins the majority of their regulatory output is an important factor for consideration in compound design. In this context, it will be important to observe whether, as seems possible, pseudokinases (like kinases) possess the inherent flexibility to succumb to mutations that interfere with drug binding while preserving their signaling function.

\section{Financial \& competing interests disclosure}

The authors gratefully acknowledge BBSRC for a DTPStudentship (to DMF) and for project grant funding BB/
N021703/1 (to DPB and PAE). The authors have no other relevant affiliations or financial involvement with any organization or entity with a financial interest in or financial conflict with the subject matter or materials discussed in the manuscript apart from those disclosed.

No writing assistance was utilized in the production of this manuscript.

\section{Open access}

This work is licensed under the Attribution-NonCommercialNoDerivatives 4.0 Unported License. To view a copy of this license, visit http://creativecommons.org/licenses/by-nc-nd/4.0/

\section{Executive summary}

- Pseudokinases are ubiquitously found in eukaryote kinomes, and are involved in a myriad of cellular processes, where they often serve as signaling modulators.

- The disruption of pseudokinase signaling function is strongly associated with a wide range of human diseases, including cancer.

- Regulated pseudokinase-dependent signaling is achieved by ligand or protein induced conformational transitions, with signal transduction closely linked to adoption of an appropriate pseudo-active state.

- Small-molecule inhibitors with the ability to target and (de)stabilize different pseudokinase structural states are promising candidates for the development of new pseudokinase-targeted therapeutics.

\section{References}

Papers of special note have been highlighted as:

- of interest; $\bullet$ of considerable interest.

1 Caenepeel S, Charydczak G, Sudarsanam S, Hunter T, Manning G. The mouse kinome: discovery and comparative genomics of all mouse protein kinases. Proc. Natl Acad. Sci. USA 101(32), 11707-11712 (2004).

2 Manning G, Whyte DB, Martinez R, Hunter T, Sudarsanam $\mathrm{S}$. The protein kinase complement of the human genome. Science 298(5600), 1912-1934 (2002).

- Annotation of the human kinome and pseudokinome.

3 Eyers PA, Murphy JM. Dawn of the dead: protein pseudokinases signal new adventures in cell biology. Biochem. Soc. Trans. 41(4), 969-974 (2013).

4 Scheeff ED, Eswaran J, Bunkoczi G, Knapp S, Manning G. Structure of the pseudokinase VRK3 reveals a degraded catalytic site, a highly conserved kinase fold, and a putative regulatory binding site. Structure 17(1), 128-138 (2009).

- Careful analysis of the pseudokinase VRK3, with essential information on human pseudokinases.

5 Manning G, Reiner DS, Lauwaet T et al. The minimal kinome of Giardia lamblia illuminates early kinase evolution and unique parasite biology. Genome Biol. 12(7), 1 (2011).

6 Wilhelm M, Schlegl J, Hahne H et al. Mass-spectrometrybased draft of the human proteome. Nature 509(7502), 582-587 (2014).

7 Hanahan D, Weinberg RA. Hallmarks of cancer: the next generation. Cell 144(5), 646-674 (2011).

8 Cohen P. Protein kinases - the major drug targets of the twenty-first century? Nat. Rev. Drug Discov. 1(4), 309-315 (2002).
9 Coghlan M, Smith D. Introduction to the Kinases in Diabetes Biochemical Society focused meeting: are protein kinases good targets for antidiabetic drugs? Biochem. Soc. Trans. 33(2), 339-342 (2005).

10 Dhalla NS, Müller AL. Protein kinases as drug development targets for heart disease therapy. Pharmaceuticals 3(7), 2111-2145 (2010).

11 Flight MH. Neurodegenerative diseases: new kinase targets for Alzheimer's disease. Nat. Rev. Drug Discov. 12(10), 739-739 (2013).

12 Mohedas AH, Xing X, Armstrong KA, Bullock AN, Cuny GD, Yu PB. Development of an ALK2-biased BMP type I receptor kinase inhibitor. ACS Chem. Biol. 8(6), 1291-1302 (2013).

13 Kantarjian H, Sawyers C, Hochhaus A et al. Hematologic and cytogenetic responses to imatinib mesylate in chronic myelogenous leukemia. N. Engl. J. Med. 346(9), 645-652 (2002).

14 Chapman PB, Hauschild A, Robert C et al. Improved survival with vemurafenib in melanoma with BRAF V600E mutation. N. Engl. J. Med. 364(26), 2507-2516 (2011).

15 Arteaga CL, Engelman JA. ERBB receptors: from oncogene discovery to basic science to mechanism-based cancer therapeutics. Cancer Cell 25(3), 282-303 (2014).

16 Eyers PA, Murphy JM. The evolving world of pseudoenzymes: proteins, prejudice and zombies. BMC Biol. 14(1), 98 (2016).

17 Boudeau J, Miranda-Saavedra D, Barton GJ, Alessi DR. Emerging roles of pseudokinases. Trends Cell Biol. 16(9), 443-452 (2006). 
18 Bailey FP, Byrne DP, Mcskimming D, Kannan N, Eyers PA. Going for broke: targeting the human cancer pseudokinome. Biochem. J. 465(2), 195-211 (2015).

19 Murphy JM, Zhang Q, Young SN et al. A robust methodology to subclassify pseudokinases based on their nucleotide-binding properties. Biochem. J. 457(2), 323-334 (2014).

-• First comprehensive analysis of binding propensity of a varied panel of pseudokinases.

20 Mukherjee K, Sharma M, Jahn R, Wahl MC, Sudhof TC. Evolution of CASK into a Mg2+-sensitive kinase. Sci. Signal. 3(119), ra33 (2010).

21 Mukherjee K, Sharma M, Urlaub H et al. CASK functions as a Mg 2+-independent neurexin kinase. Cell 133(2), 328-339 (2008).

22 Bailey FP, Byrne DP, Oruganty K et al. The Tribbles 2 (TRB2) pseudokinase binds to ATP and autophosphorylates in a metalindependent manner. Biochem. J. 467(1), 47-62 (2015).

23 Babon JJ, Lucet IS, Murphy JM, Nicola NA, Varghese LN. The molecular regulation of Janus kinase (JAK) activation. Biochem. J. 462(1), 1-13 (2014).

24 Shi F, Telesco SE, Liu Y, Radhakrishnan R, Lemmon MA. ErbB3/HER3 intracellular domain is competent to bind ATP and catalyze autophosphorylation. Proc. Natl Acad. Sci. USA 107(17), 7692-7697 (2010).

25 Xie T, Lim SM, Westover KD et al. Pharmacological targeting of the pseudokinase Her3. Nat. Chem. Biol. 10(12), 1006-1012 (2014).

- Covalent targeting of HER3 with small-molecule ligands.

26 Xu B-E, English JM, Wilsbacher JL, Stippec S, Goldsmith EJ, Cobb MH. WNK1, a novel mammalian serine/threonine protein kinase lacking the catalytic lysine in subdomain II. J. Biol. Chem. 275(22), 16795-16801 (2000).

27 Zeqiraj E, Filippi BM, Goldie S et al. ATP and MO25 $\alpha$ regulate the conformational state of the STRAD $\alpha$ pseudokinase and activation of the LKB1 tumour suppressor. PLoS Biol. 7(6), e1000126 (2009).

28 Murphy JM, Lucet IS, Hildebrand JM et al. Insights into the evolution of divergent nucleotide-binding mechanisms among pseudokinases revealed by crystal structures of human and mouse MLKL. Biochem. J. 457(3), 369-377 (2014).

29 Brennan DF, Dar AC, Hertz NT et al. A Raf-induced allosteric transition of KSR stimulates phosphorylation of MEK. Nature 472(7343), 366-369 (2011).

30 Reiterer V, Eyers PA, Farhan H. Day of the dead: pseudokinases and pseudophosphatases in physiology and disease. Trends Cell Biol. 24(9), 489-505 (2014).

31 Jura N, Shan Y, Cao X, Shaw DE, Kuriyan J. Structural analysis of the catalytically inactive kinase domain of the human EGF receptor 3. Proc. Natl Acad. Sci. USA 106(51), 21608-21613 (2009).

32 Kung JE, Jura N. Structural basis for the non-catalytic functions of protein kinases. Structure 24(1), 7-24 (2016).

33 Laurence A, Pesu M, Silvennoinen O, O'shea J. JAK kinases in health and disease: an update. Open Rheumatol. J. 6, 232-244 (2012).
34 Haan C, Behrmann I, Haan S. Perspectives for the use of structural information and chemical genetics to develop inhibitors of Janus kinases. J. Cell. Mol. Med. 14(3), 504-527 (2010).

35 Ungureanu D, Wu J, Pekkala T et al. The pseudokinase domain of JAK2 is a dual-specificity protein kinase that negatively regulates cytokine signaling. Nat. Struct. Mol. Biol. 18(9), 971-976 (2011).

Saharinen P, Takaluoma K, Silvennoinen O. Regulation of the Jak2 tyrosine kinase by its pseudokinase domain. Mol. Cell. Biol. 20 (10), 3387-3395 (2000).

37 Varghese LN, Ungureanu D, Liau NP et al. Mechanistic insights into activation and SOCS3-mediated inhibition of myeloproliferative neoplasm-associated JAK2 mutants from biochemical and structural analyses. Biochem. J. 458(2), 395-405 (2014).

38 Min X, Ungureanu D, Maxwell S et al. Structural and functional characterization of the $\mathrm{JH} 2$ pseudokinase domain of JAK family tyrosine kinase 2 (TYK2). J. Biol. Chem. 290(45), 27261-27270 (2015).

39 Vainchenker W, Constantinescu S. JAK/STAT signaling in hematological malignancies. Oncogene 32(21), 2601-2613 (2013).

40 Saharinen P, Silvennoinen O. The pseudokinase domain is required for suppression of basal activity of Jak 2 and Jak3 tyrosine kinases and for cytokine-inducible activation of signal transduction. J. Biol. Chem. 277(49), 47954-47963 (2002).

41 Kralovics R, Passamonti F, Buser AS et al. A gain-of-function mutation of JAK2 in myeloproliferative disorders. N. Engl. J. Med. 352(17), 1779-1790 (2005).

42 James C, Ugo V, Le Couédic J-P et al. A unique clonal JAK2 mutation leading to constitutive signalling causes polycythaemia vera. Nature 434(7037), 1144-1148 (2005).

43 Levine RL, Wadleigh M, Cools J et al. Activating mutation in the tyrosine kinase JAK2 in polycythemia vera, essential thrombocythemia, and myeloid metaplasia with myelofibrosis. Cancer Cell 7(4), 387-397 (2005).

44 Baxter EJ, Scott LM, Campbell PJ et al. Acquired mutation of the tyrosine kinase JAK2 in human myeloproliferative disorders. Lancet 365(9464), 1054-1061 (2005).

45 Scott LM, Tong W, Levine RL et al. JAK2 exon 12 mutations in polycythemia vera and idiopathic erythrocytosis. N. Engl. J. Med. 356(5), 459-468 (2007).

46 Lu X, Huang LJ-S, Lodish HF. Dimerization by a cytokine receptor is necessary for constitutive activation of JAK2V617F. J. Biol. Chem. 283(9), 5258-5266 (2008).

47 Dusa A, Mouton C, Pecquet C, Herman M, Constantinescu SN. JAK2 V617F constitutive activation requires JH2 residue F595: a pseudokinase domain target for specific inhibitors. PLoS ONE 5(6), e11157 (2010).

48 Hammarén HM, Ungureanu D, Grisouard J, Skoda RC, Hubbard SR, Silvennoinen O. ATP binding to the pseudokinase domain of JAK2 is critical for pathogenic activation. Proc. Natl Acad. Sci. USA 112(15), 4642-4647 (2015). 
49 Mascarenhas J, Hoffman R. Ruxolitinib: the first FDA approved therapy for the treatment of myelofibrosis. Clin. Cancer. Res. 18(11), 3008-3014 (2012).

50 Verstovsek S, Vannucchi AM, Griesshammer M et al. Ruxolitinib versus best available therapy in patients with polycythemia vera: 80 -week follow-up from the RESPONSE trial. Haematologica 101(7), 821-829 (2016).

51 Novotny CJ, Pollari S, Park JH, Lemmon MA, Shen W, Shokat KM. Overcoming resistance to HER2 inhibitors through state-specific kinase binding. Nat. Chem. Biol. 12(11), 923-930 (2016).

52 Yarden Y, Sliwkowski MX. Untangling the ErbB signalling network. Nat. Rev. Mol. Cell Biol. 2(2), 127-137 (2001).

53 Zhang N, Chang Y, Rios A, An Z. HER3/ErbB3, an emerging cancer therapeutic target. Acta Biochim. Biophys. Sin. (Shanghai) 48(1), 39-48 (2016).

54 Vaught DB, Stanford JC, Young C et al. HER3 is required for HER2-induced preneoplastic changes to the breast epithelium and tumor formation. Cancer Res. 72(10), 2672-2682 (2012).

55 Jaiswal BS, Kljavin NM, Stawiski EW et al. Oncogenic ERBB3 mutations in human cancers. Cancer Cell 23(5), 603-617 (2013).

56 Sithanandam G, Anderson LM. The ERBB3 receptor in cancer and cancer gene therapy. Cancer Gene Ther. 15(7), 413-448 (2008).

57 Naidu R, Yadav M, Nair S, Kutty MK. Expression of c-erbB3 protein in primary breast carcinomas. Br. J. Cancer 78(10), 1385-1390 (1998).

58 Ma J, Lyu H, Huang J, Liu B. Targeting of erbB3 receptor to overcome resistance in cancer treatment. Mol. Cancer 13(1), 1 (2014).

59 Huang S, Li C, Armstrong EA et al. Dual targeting of EGFR and HER3 with MEHD7945A overcomes acquired resistance to EGFR inhibitors and radiation. Cancer Res. 73(2), 824-833 (2013).

60 Umelo I, Noeparast A, Chen G et al. Identification of a novel HER3 activating mutation homologous to EGFR-L858R in lung cancer. Oncotarget 7(3), 3068 (2016).

61 Littlefield P, Liu L, Mysore V, Shan Y, Shaw DE, Jura N. Structural analysis of the EGFR/HER3 heterodimer reveals the molecular basis for activating HER3 mutations. Sci. Signal. 7(354), ra114 (2014).

62 Zeqiraj E, Filippi BM, Deak M, Alessi DR, Van Aalten DM. Structure of the LKB1-STRAD-MO25 complex reveals an allosteric mechanism of kinase activation. Science 326(5960), 1707-1711 (2009).

-• Landmark structural analysis of an allosteric nucleotidebinding pseudokinase in complex with a canonical kinase and cofactor protein.

63 Lizcano JM, Göransson O, Toth R et al. LKB1 is a master kinase that activates 13 kinases of the AMPK subfamily, including MARK/PAR-1. EMBO J. 23(4), 833-843 (2004).

64 Boudeau J, Scott JW, Resta N et al. Analysis of the LKB1STRAD-MO25 complex. J. Cell Sci. 117(Pt 26), 6365-6375 (2004).
65 Huang X, Wullschleger S, Shpiro N et al. Important role of the LKB1-AMPK pathway in suppressing tumorigenesis in PTEN-deficient mice. Biochem. J. 412(2), 211-221 (2008).

66 Roy F, Laberge G, Douziech M, Ferland-Mccollough D, Therrien M. KSR is a scaffold required for activation of the ERK/MAPK module. Genes Dev. 16(4), 427-438 (2002).

67 Nguyen A, Burack WR, Stock JL et al. Kinase suppressor of Ras (KSR) is a scaffold which facilitates mitogen-activated protein kinase activation in vivo. Mol. Cell. Biol. 22(9), 3035-3045 (2002).

68 Zhang H, Koo CY, Stebbing J, Giamas G. The dual function of KSR1: a pseudokinase and beyond. Biochem. Soc. Trans. 41(4), 1078-1082 (2013).

69 Goettel JA, Liang D, Hilliard VC et al. KSR1 is a functional protein kinase capable of serine autophosphorylation and direct phosphorylation of MEK1. Exp. Cell Res. 317(4), 452-463 (2011).

70 Xing HR, Kolesnick R. Kinase suppressor of Ras signals through Thr269 of c-Raf-1. J. Biol. Chem. 276(13), 9733-9741 (2001).

71 Zafrullah M, Yin X, Haimovitz-Friedman A, Fuks Z, Kolesnick R. Kinase suppressor of Ras transphosphorylates c-Raf-1. Biochem. Biophys. Res. Commun. 390(3), 434-440 (2009).

72 Stewart S, Sundaram M, Zhang Y, Lee J, Han M, Guan K-L. Kinase suppressor of Ras forms a multiprotein signaling complex and modulates MEK localization. Mol. Cell. Biol. 19(8), 5523-5534 (1999).

73 Kortum RL, Lewis RE. The molecular scaffold KSR1 regulates the proliferative and oncogenic potential of cells. Mol. Cell. Biol. 24(10), 4407-4416 (2004).

74 Xing HR, Cordon-Cardo C, Deng X et al. Pharmacologic inactivation of kinase suppressor of ras-1 abrogates Rasmediated pancreatic cancer. Nat. Med. 9(10), 1267-1268 (2003).

75 Pearce LR, Atanassova N, Banton MC et al. KSR2 mutations are associated with obesity, insulin resistance, and impaired cellular fuel oxidation. Cell 155(4), 765-777 (2013).

76 Murphy JM, Czabotar PE, Hildebrand JM et al. The pseudokinase MLKL mediates necroptosis via a molecular switch mechanism. Immunity 39(3), 443-453 (2013).

77 Sun L, Wang H, Wang Z et al. Mixed lineage kinase domain-like protein mediates necrosis signaling downstream of RIP3 kinase. Cell 148(1), 213-227 (2012).

78 Su L, Quade B, Wang H, Sun L, Wang X, Rizo J. A plug release mechanism for membrane permeation by MLKL. Structure 22(10), 1489-1500 (2014).

79 Vandenabeele P, Galluzzi L, Vanden Berghe T, Kroemer G. Molecular mechanisms of necroptosis: an ordered cellular explosion. Nat. Rev. Mol. Cell Biol. 11(10), 700-714 (2010).

80 Alvarez-Diaz S, Dillon CP, Lalaoui N et al. The pseudokinase MLKL and the kinase RIPK3 have distinct roles in autoimmune disease caused by loss of death-receptorinduced apoptosis. Immunity 45(3), 513-526 (2016).

81 Forbes S, Bhamra G, Bamford S et al. The catalogue of somatic mutations in cancer (COSMIC). Curr. Protoc. Hum. Genet. Chapter 10, Unit 10.11 (2008). 
82 Foulkes DM, Byrne DP, Bailey FP, Eyers PA. Tribbles pseudokinases: novel targets for chemical biology and drug discovery? Biochem. Soc. Trans. 43(5), 1095-1103 (2015).

83 Eyers PA, Keeshan K, Kannan N. Tribbles in the 21st Century: the evolving roles of tribbles pseudokinases in biology and disease. Trends Cell Biol. doi:10.1016/j. tcb.2016.11.002 (2016) (Epub ahead of print).

84 Iwamoto S, Boonvisut S, Makishima S, Ishizuka Y, Watanabe K, Nakayama K. The role of TRIB1 in lipid metabolism; from genetics to pathways. Biochem. Soc. Trans. 43(5), 1063-1068 (2015).

85 Johnston J, Basatvat S, Ilyas Z, Francis S, Kiss-Toth E. Tribbles in inflammation. Biochem. Soc. Trans. 43(5), 1069-1074 (2015).

Lohan F, Keeshan K. The functionally diverse roles of tribbles. Biochem. Soc. Trans. 41(4), 1096-1100 (2013). Yokoyama T, Nakamura T. Tribbles in disease: signaling pathways important for cellular function and neoplastic transformation. Cancer Sci. 102(6), 1115-1122 (2011).

88 Murphy JM, Nakatani Y, Jamieson SA, Dai W, Lucet IS, Mace PD. Molecular mechanism of CCAAT-enhancer binding protein recruitment by the TRIB1 pseudokinase. Structure 23(11), 2111-2121 (2015).

- Structural analysis of human Tribbles 1 , a pseudokinase that functions as a potential platform for substrate ubiquitylation.

89 Keeshan K, He Y, Wouters BJ et al. Tribbles homolog 2 inactivates $\mathrm{C} / \mathrm{EBPalpha}$ and causes acute myelogenous leukemia. Cancer Cell 10 (5), 401-411 (2006).

90 Storlazzi CT, Fioretos T, Paulsson K et al. Identification of a commonly amplified $4.3 \mathrm{Mb}$ region with overexpression of $\mathrm{C} 8 \mathrm{FW}$, but not MYC in MYC-containing double minutes in myeloid malignancies. Hum. Mol. Genet. 13(14), 1479-1485 (2004).

91 Grandinetti KB, Stevens TA, Ha S et al. Overexpression of TRIB2 in human lung cancers contributes to tumorigenesis through downregulation of C/EBPalpha. Oncogene 30 (30), 3328-3335 (2011).

92 Wang J, Park JS, Wei Y et al. TRIB2 acts downstream of Wnt/TCF in liver cancer cells to regulate YAP and C/ EBPalpha function. Mol. Cell 51(2), 211-225 (2013).

93 Zanella F, Renner O, Garcia B et al. Human TRIB2 is a repressor of FOXO that contributes to the malignant phenotype of melanoma cells. Oncogene 29(20), 2973-2982 (2010).

94 Yokoyama T, Toki T, Aoki Y et al. Identification of TRIB1 R107L gain-of-function mutation in human acute megakaryocytic leukemia. Blood 119(11), 2608-2611 (2012).

95 Qi L, Heredia JE, Altarejos JY et al. TRB3 links the E3 ubiquitin ligase COP1 to lipid metabolism. Science 312(5781), 1763-1766 (2006).

96 Zhang W, Liu J, Tian L, Liu Q, Fu Y, Garvey WT. TRIB3 mediates glucose-induced insulin resistance via a mechanism that requires the hexosamine biosynthetic pathway. Diabetes 62(12), 4192-4200 (2013).

97 Tang BL. (WNK)ing at death: with-no-lysine (Wnk) kinases in neuropathies and neuronal survival. Brain Res. Bull. 125, 92-98 (2016).
98 Piala AT, Moon TM, Akella R, He H, Cobb MH, Goldsmith EJ. Chloride sensing by WNK1 involves inhibition of autophosphorylation. Sci. Signal. 7(324), ra41 (2014).

99 Wilson FH, Disse-Nicodeme S, Choate KA et al. Human hypertension caused by mutations in WNK kinases. Science 293(5532), 1107-1112 (2001).

100 Sun X, Gao L, Yu RK, Zeng G. Down-regulation of WNK1 protein kinase in neural progenitor cells suppresses cell proliferation and migration. J. Neurochem. 99(4), 1114-1121 (2006).

101 Zhu W, Begum G, Pointer K et al. WNK1-OSR1 kinase-mediated phospho-activation of $\mathrm{Na}+-\mathrm{K}+-2 \mathrm{Cl}-$ cotransporter facilitates glioma migration. Mol. Cancer 13, 31 (2014).

102 Haas BR, Cuddapah VA, Watkins S, Rohn KJ, Dy TE, Sontheimer H. With-no-lysine kinase 3 (WNK3) stimulates glioma invasion by regulating cell volume. Am. J. Physiol. Cell Physiol. 301(5), C1150-C1160 (2011).

103 Moniz S, Matos P, Jordan P. WNK2 modulates MEK1 activity through the Rho GTPase pathway. Cell. Signal. 20(10), 1762-1768 (2008).

104 Zhang J, Yang PL, Gray NS. Targeting cancer with small molecule kinase inhibitors. Nat. Rev. Cancer 9(1), 28-39 (2009)

105 Eyers PA, Craxton M, Morricel N, Cohen P, Goedert M. Conversion of SB 203580-insensitive MAP kinase family members to drug-sensitive forms by a single amino-acid substitution. Chem. Biol. 5(6), 321-328 (1998).

106 Eyers PA, Van Den IP, Quinlan RA, Goedert M, Cohen P. Use of a drug-resistant mutant of stress-activated protein kinase $2 \mathrm{a} / \mathrm{p} 38$ to validate the in vivo specificity of SB 203580. FEBS Lett. 451(2), 191-196 (1999).

107 Knight ZA, Lin H, Shokat KM. Targeting the cancer kinome through polypharmacology. Nat. Rev. Cancer 10(2), 130-137 (2010).

108 Bishop AC, Ubersax JA, Petsch DT et al. A chemical switch for inhibitor-sensitive alleles of any protein kinase. Nature 407(6802), 395-401 (2000).

109 Bailey FP, Andreev VI, Eyers PA. The resistance tetrad: amino acid hotspots for kinome-wide exploitation of drugresistant protein kinase alleles. Methods Enzymol. 548, 117-146 (2014).

110 Hammarén HM, Virtanen AT, Silvennoinen O. Nucleotidebinding mechanisms in pseudokinases. Biosci. Rep. 36(1), e00282 (2016).

111 Littlefield P, Moasser MM, Jura N. An ATP-competitive inhibitor modulates the allosteric function of the HER3 pseudokinase. Chem. Biol. 21(4), 453-458 (2014).

- The promiscuous kinase inhibitor bosutinib stabilizes an 'activating' confirmation of HER3 pseudokinase.

112 Davis MI, Hunt JP, Herrgard S et al. Comprehensive analysis of kinase inhibitor selectivity. Nat. Biotechnol. 29(11), 1046-1051 (2011).

113 Claus J, Cameron AJ, Parker PJ. Pseudokinase drug intervention: a potentially poisoned chalice. Biochem. Soc. Trans. 41(4), 1083-1088 (2013). 
114 Hatzivassiliou G, Song K, Yen I et al. RAF inhibitors prime wild-type RAF to activate the MAPK pathway and enhance growth. Nature 464(7287), 431-435 (2010).

115 Poulikakos PI, Zhang C, Bollag G, Shokat KM, Rosen N. RAF inhibitors transactivate RAF dimers and ERK signalling in cells with wild-type BRAF. Nature 464(7287), 427-430 (2010).

116 Tokarski JS, Zupa-Fernandez A, Tredup JA et al. Tyrosine kinase 2-mediated signal transduction in $\mathrm{T}$ lymphocytes is blocked by pharmacological stabilization of its pseudokinase domain. J. Biol. Chem. 290(17), 11061-11074 (2015).

117 Liu Y, Gray NS. Rational design of inhibitors that bind to inactive kinase conformations. Nat. Chem. Biol. 2(7), 358-364 (2006).

118 Manley P, Cowan-Jacob S, Buchdunger E et al. Imatinib: a selective tyrosine kinase inhibitor. Eur. J. Cancer 38, S19-S27 (2002).

119 Nagar B, Bornmann WG, Pellicena P et al. Crystal structures of the kinase domain of c-Abl in complex with the small molecule inhibitors PD173955 and imatinib (STI-571). Cancer Res. 62(15), 4236-4243 (2002).

120 Druker BJ, Talpaz M, Resta DJ et al. Efficacy and safety of a specific inhibitor of the BCR-ABL tyrosine kinase in chronic myeloid leukemia. N. Engl. J. Med. 2001(344), 1031-1037 (2001).

121 O'brien SG, Guilhot F, Larson RA et al. Imatinib compared with interferon and low-dose cytarabine for newly diagnosed chronic-phase chronic myeloid leukemia. N. Engl. J. Med. 348(11), 994-1004 (2003).

122 Taylor SS, Shaw A, Hu J, Meharena HS, Kornev A. Pseudokinases from a structural perspective. Biochem. Soc. Trans. 41(4), 981-986 (2013).

123 Han Y, Donovan J, Rath S, Whitney G, Chitrakar A, Korennykh A. Structure of human RNase L reveals the basis for regulated RNA decay in the IFN response. Science 343(6176), 1244-1248 (2014).

124 Huang H, Zeqiraj E, Dong B et al. Dimeric structure of pseudokinase RNase $\mathrm{L}$ bound to $2-5 \mathrm{~A}$ reveals a basis for interferon-induced antiviral activity. Mol. Cell 53(2), 221-234 (2014).

125 Dong B, Silverman RH. Alternative function of a protein kinase homology domain in 2',5'-oligoadenylate dependent RNase L. Nucleic Acids Res. 27(2), 439-445 (1999).

126 Garner AP, Bialucha CU, Sprague ER et al. An antibody that locks HER3 in the inactive conformation inhibits tumor growth driven by HER2 or neuregulin. Cancer Res. 73(19), 6024-6035 (2013).

127 Agus DB, Akita RW, Fox WD et al. Targeting ligandactivated ErbB2 signaling inhibits breast and prostate tumor growth. Cancer Cell 2(2), 127-137 (2002).

128 Dhawan NS, Scopton AP, Dar AC. Small molecule stabilization of the KSR inactive state antagonizes oncogenic Ras signalling. Nature 537(7618 ), 112-116 (2016).

129 Hildebrand JM, Tanzer MC, Lucet IS et al. Activation of the pseudokinase MLKL unleashes the four-helix bundle domain to induce membrane localization and necroptotic cell death. Proc. Natl Acad. Sci. USA 111(42), 15072-15077 (2014).
130 Ma B, Marcotte D, Paramasivam M et al. ATP-competitive MLKL binders have no functional impact on necroptosis. PLoS ONE 11(11), e0165983 (2016).

131 Gustafson WC, Meyerowitz JG, Nekritz EA et al. Drugging MYCN through an allosteric transition in Aurora kinase A. Cancer Cell 26(3), 414-427 (2014).

132 Dodson CA, Kosmopoulou M, Richards MW et al. Crystal structure of an Aurora-A mutant that mimics Aurora-B bound to MLN8054: insights into selectivity and drug design. Biochem. J. 427(1), 19-28 (2010).

133 Barf T, Kaptein A. Irreversible protein kinase inhibitors: balancing the benefits and risks. J. Med. Chem 55(14), 6243-6262 (2012).

134 Cowan-Jacob SW, Jahnke W, Knapp S. Novel approaches for targeting kinases: allosteric inhibition, allosteric activation and pseudokinases. Future Med. Chem. 6(5), 541-561 (2014).

135 Zhang J, Adrián FJ, Jahnke W et al. Targeting Bcr-Abl by combining allosteric with ATP-binding-site inhibitors. Nature 463(7280), 501-506 (2010).

136 Lindsley CW, Zhao Z, Leister WH et al. Allosteric Akt (PKB) inhibitors: discovery and SAR of isozyme selective inhibitors. Bioorg. Med. Chem. Lett. 15(3), 761-764 (2005).

137 Wu W-I, Voegtli WC, Sturgis HL, Dizon FP, Vigers GP, Brandhuber BJ. Crystal structure of human AKT1 with an allosteric inhibitor reveals a new mode of kinase inhibition. PLoS ONE 5(9), e12913 (2010).

138 Schulze JO, Saladino G, Busschots K et al. Bidirectional allosteric communication between the ATP-binding site and the regulatory PIF Pocket in PDK1 protein kinase. Cell Chem. Biol. 23(10), 1193-1205 (2016).

139 Stefely JA, Reidenbach AG, Ulbrich A et al. Mitochondrial ADCK3 employs an atypical protein kinase-like fold to enable coenzyme Q biosynthesis. Mol. Cell 57(1), 83-94 (2015).

140 Fukuda K, Gupta S, Chen K, Wu C, Qin J. The pseudoactive site of ILK is essential for its binding to alpha-Parvin and localization to focal adhesions. Mol. Cell 36(5), 819-830 (2009).

141 Toms AV, Deshpande A, Mcnally R et al. Structure of a pseudokinase-domain switch that controls oncogenic activation of Jak kinases. Nat. Struct. Mol. Biol. 20(10), 1221-1223 (2013).

142 Bandaranayake RM, Ungureanu D, Shan Y, Shaw DE, Silvennoinen O, Hubbard SR. Crystal structures of the JAK2 pseudokinase domain and the pathogenic mutant V617F. Nat. Struct. Mol. Biol. 19(8), 754-759 (2012).

143 Leroy E, Dusa A, Colau D et al. Uncoupling JAK2 V617F activation from cytokine-induced signalling by modulation of JH2 alphaC helix. Biochem. J. 473(11), 1579-1591 (2016).

144 Xie T, Peng W, Yan C, Wu J, Gong X, Shi Y. Structural insights into RIP3-mediated necroptotic signaling. Cell Rep. 5(1), 70-78 (2013).

145 Christie M, Boland A, Huntzinger E, Weichenrieder O, Izaurralde E. Structure of the PAN3 pseudokinase reveals the basis for interactions with the PAN2 deadenylase and the GW182 proteins. Mol. Cell 51(3), 360-373 (2013). 
146 Artim SC, Mendrola JM, Lemmon MA. Assessing the range of kinase autoinhibition mechanisms in the insulin receptor family. Biochem. J. 448(2), 213-220 (2012).

147 Hackman JP, Vihola AK, Udd AB. The role of titin in muscular disorders. Ann. Med. 35(6), 434-441 (2003).

148 Mayans O, Van Der Ven PF, Wilm M et al. Structural basis for activation of the titin kinase domain during myofibrillogenesis. Nature 395(6705), 863-869 (1998).

149 Bogomolovas J, Gasch A, Simkovic F, Rigden DJ, Labeit S, Mayans $\mathrm{O}$. Titin kinase is an inactive pseudokinase scaffold that supports MuRF1 recruitment to the sarcomeric M-line. Open Biol. 4(5), 140041 (2014).

150 Leproult E, Barluenga S, Moras D, Wurtz J-M, Winssinger $\mathrm{N}$. Cysteine mapping in conformationally distinct kinase nucleotide binding sites: application to the design of selective covalent inhibitors. J. Med. Chem. 54(5), 1347-1355 (2011).

151 Wu S, Luo H, Wang H, Zhao W, Hu Q, Yang Y. Cysteinome: the first comprehensive database for proteins with targetable cysteine and their covalent inhibitors. Biochem. Biophys. Res. Commun. 478(3), 1268-1273 (2016).

152 Bandyopadhyay A, Gao J. Targeting biomolecules with reversible covalent chemistry. Curr. Opin. Chem. Biol. 34, 110-116 (2016).

153 Brown JR. Ibrutinib (PCI-32765), the first BTK (Bruton's tyrosine kinase) inhibitor in clinical trials. Curr. Hematol. Malig. Rep. 8(1), 1-6 (2013).

154 Byrd JC, Harrington B, O'brien S et al. Acalabrutinib (ACP196) in relapsed chronic lymphocytic leukemia. N. Engl. J. Med. 374(4), 323-332 (2016).

155 Humphries KM, Deal MS, Taylor SS. Enhanced dephosphorylation of cAMP-dependent protein kinase by oxidation and thiol modification. J. Biol. Chem. 280(4), 2750-2758 (2005).

156 Beullens M, Vancauwenbergh S, Morrice N et al. Substrate specificity and activity regulation of protein kinase MELK. J. Biol. Chem. 280(48), 40003-40011 (2005).

157 Liu Q, Sabnis Y, Zhao Z et al. Developing irreversible inhibitors of the protein kinase cysteinome. Chem. Biol. 20(2), 146-159 (2013).

- Landmark review discussing the complexity of the cysteinome in the human kinome.

158 Mah R, Thomas JR, Shafer CM. Drug discovery considerations in the development of covalent inhibitors. Bioorg. Med. Chem. Lett. 24(1), 33-39 (2014).

159 Lanning BR, Whitby LR, Dix MM et al. A road map to evaluate the proteome-wide selectivity of covalent kinase inhibitors. Nat. Chem. Biol. 10(9), 760-767 (2014).

160 Honigberg LA, Smith AM, Sirisawad M et al. The Bruton tyrosine kinase inhibitor PCI-32765 blocks B-cell activation and is efficacious in models of autoimmune disease and B-cell malignancy. Proc. Natl Acad. Sci. USA 107(29), 13075-13080 (2010).

161 Byrd JC, Furman RR, Coutre SE et al. Targeting BTK with ibrutinib in relapsed chronic lymphocytic leukemia. N. Engl. J. Med. 369(1), 32-42 (2013).
162 Wang ML, Rule S, Martin P et al. Targeting BTK with ibrutinib in relapsed or refractory mantle-cell lymphoma. $N$. Engl. J. Med. 369(6), 507-516 (2013).

163 Goedken ER, Argiriadi MA, Banach DL et al. Tricyclic covalent inhibitors selectively target Jak 3 through an active site thiol. J. Biol. Chem. 290(8), 4573-4589 (2015).

164 Dubovsky JA, Beckwith KA, Natarajan G et al. Ibrutinib is an irreversible molecular inhibitor of ITK driving a Th1-selective pressure in T lymphocytes. Blood 122(15), 2539-2549 (2013).

165 Kung A, Chen Y-C, Schimpl M et al. Development of specific, irreversible inhibitors for a receptor tyrosine kinase EphB3. J. Am. Chem. Soc. 138(33), 10554-10560 (2016).

166 Zhang $\mathrm{T}$, Inesta-Vaquera $\mathrm{F}$, Niepel $\mathrm{M}$ et al. Discovery of potent and selective covalent inhibitors of JNK. Chem. Biol. 19(1), 140-154 (2012).

167 Hagel M, Miduturu C, Sheets M et al. First selective small molecule inhibitor of FGFR4 for the treatment of hepatocellular carcinomas with an activated FGFR4 signaling pathway. Cancer Discov. 5(4), 424-437 (2015).

168 Zhou W, Hur W, Mcdermott U et al. A structure-guided approach to creating covalent FGFR inhibitors. Chem. Biol. 17(3), 285-295 (2010).

169 Cohen MS, Zhang C, Shokat KM, Taunton J. Structural bioinformatics-based design of selective, irreversible kinase inhibitors. Science 308(5726), 1318-1321 (2005).

170 Henise JC, Taunton J. Irreversible Nek2 kinase inhibitors with cellular activity. J. Med. Chem. 54(12), 4133-4146 (2011).

171 Amin DN, Campbell MR, Moasser MM. The role of HER3, the unpretentious member of the HER family, in cancer biology and cancer therapeutics. Semin. Cell Dev. Biol. 21(9), 944-950 (2010).

172 Schirmer A, Kennedy J, Murli S, Reid R, Santi DV. Targeted covalent inactivation of protein kinases by resorcylic acid lactone polyketides. Proc. Natl Acad. Sci. USA 103(11), 4234-4239 (2006).

173 Wissner A, Floyd MB, Johnson BD et al. 2-(Quinazolin4-ylamino)-[1, 4] benzoquinones as covalent-binding, irreversible inhibitors of the kinase domain of vascular endothelial growth factor receptor-2. J. Med. Chem. 48(24), 7560-7581 (2005).

174 Rossi A, Kapahi P, Natoli G et al. Anti-inflammatory cyclopentenone prostaglandins are direct inhibitors of I $\mathrm{B}$ kinase. Nature 403(6765), 103-118 (2000).

175 Humphries KM, Pennypacker JK, Taylor SS. Redox regulation of cAMP-dependent protein kinase signaling: kinase versus phosphatase inactivation. J. Biol. Chem. 282(30), 22072-22079 (2007).

176 Mcskimming DI, Dastgheib S, Baffi TR et al. KinView: a visual comparative sequence analysis tool for integrated kinome research. Mol. Biosyst. 12(12), 3651-3665 (2016).

177 Wang Y, Ho TG, Bertinetti D et al. Isoform-selective disruption of AKAP-localized PKA using hydrocarbon stapled peptides. ACS Chem. Biol. 9(3), 635-642 (2014).

178 Xie X, Gao L, Shull AY, Teng Y. Stapled peptides: providing the best of both worlds in drug development. Future Med. Chem. 8(16), 1969-1980 (2016). 